\title{
Magnetic frequency response of High-Luminosity Large Hadron Collider beam screens
}

\author{
M. Morrone,${ }^{1,2, *}$ M. Martino, ${ }^{1}$ R. De Maria, ${ }^{1}$ M. Fitterer, ${ }^{3}$ and C. Garion ${ }^{1}$ \\ ${ }^{1}$ European Organization for Nuclear Research (CERN), Espl. des Particules 1, 1211 Geneva, Switzerland \\ ${ }^{2}$ Department of Mechanical Engineering, Imperial College London, \\ Exhibition Road, South Kensington Campus, London SW7 2AZ, United Kingdom \\ ${ }^{3}$ Fermi National Accelerator Laboratory (FNAL), Pine Street and Kirk Rd, Batavia, Illinois 60510, USA
}

(Received 14 August 2018; published 23 January 2019)

\begin{abstract}
Magnetic fields used to control particle beams in accelerators are usually controlled by regulating the electrical current of the power converters. In order to minimize lifetime degradation and ultimately luminosity loss in circular colliders, current-noise is a highly critical figure of merit of power converters, in particular for magnets located in areas with high beta-function, like the High-Luminosity Large Hadron Collider (HL-LHC) insertions. However, what is directly acting upon the beam is the magnetic field and not the current of the power converter, which undergoes several frequency-dependent transformations until the desired magnetic field, seen by the beam, is obtained. Beam screens are very rarely considered when assessing or specifying the noise figure of merit, but their magnetic frequency response is such that they realize relatively effective low pass filtering of the magnetic field produced by the system magnet-power converter. This work aims at filling this gap by quantifying the expected impact of different beam screen layouts for the most relevant HL-LHC insertion magnets. A well-defined postprocessing technique is used to derive the frequency response of the different multipoles from multiphysics finite element method (FEM) simulation results. In addition, a wellapproximated analytical formula for the low-frequency range of multi-layered beam screens is presented.
\end{abstract}

DOI: 10.1103/PhysRevAccelBeams.22.013501

\section{INTRODUCTION}

In the framework of the High-Luminosity Large Hadron Collider (HL-LHC) project, numerous components of the accelerator will be upgraded during the third LHC long shutdown [1,2]. The main focus lies on the interaction region (IR) of LHC Point 1, where the ATLAS experiment is located, and Point 5 occupied by the CMS detector. The layout, for either side of Point 1 or Point 5, is depicted in Fig. 1. New beam screens will be installed inside the so called inner triplet (IT) quadrupole magnets, namely Q1, Q2a, Q2b, and Q3 (see Fig. 2), together with the separation-recombination dipoles D1 and D2 [3].

All these magnets are installed at locations with high beta function and the beam is therefore particularly sensitive to any changes in the magnetic field of these magnets. The impact of the field fluctuations due to power supply noise, or the so called ripple, on the beam lifetime has been studied in the past in particular at the Super Proton

\footnotetext{
*Corresponding author. marco.morrone@cern.ch

Published by the American Physical Society under the terms of the Creative Commons Attribution 4.0 International license. Further distribution of this work must maintain attribution to the author(s) and the published article's title, journal citation, and DOI.
}

Synchrotron (SPS) at CERN [4-7], the Hadron Electron Ring Facility (HERA) at DESY $[8,9]$ and the TEVATRON at FERMILAB [10]. In the case of the SPS a tune ripple of $10^{-4}$ turned out to be acceptable while experiences at HERA showed that a tune ripple of $10^{-5}$ for low frequencies and $10^{-4}$ for high frequencies could lead to a significant decrease in lifetime. Typically, a few distinct frequencies feature much higher amplitudes and therefore, the focus of these studies was also to highlight the impact of a few distinct frequency lines. It was derived theoretically [8] as well as proven experimentally [4,5] that several frequencies in the noise spectrum are significantly more harmful than a single one. This knowledge was then applied to the LHC [11] and more recently to the HL-LHC [12-14] resulting in strict tolerances for the noise generated by the power converters. The HL-LHC studies revealed that a tune modulation due to power converter ripple in the quadrupoles in the range of $10^{-5}$ to $10^{-6}$ for specific frequencies lead to a visible decrease of the dynamic aperture and thus an expected degradation of the beam lifetime, see Fig. 3 . Those studies approximated the magnet circuit with a simple RL-series circuit, but did not include the impact of the beam screen shielding that could change the figures on power converter noise tolerances. The minimization of the tune modulation to such small values, especially for the HL-LHC IT and separation dipoles where $\beta$-functions reach 


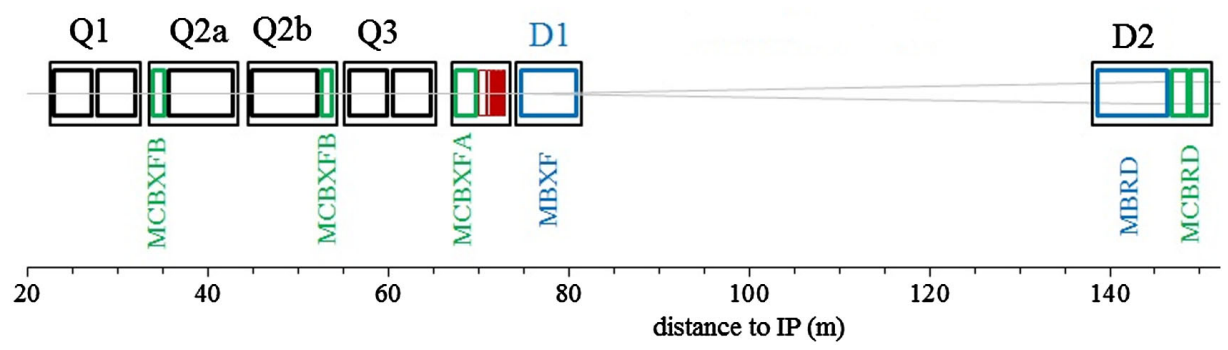

FIG. 1. The new layout of the right side of the region close to Point 1 and Point 5 foreseen for the HL-LHC. The experiment is on the left side of the figure (not shown) and the rest of the ring continues on the right. The left side of the layout is symmetric with respect to the interaction point.

about $20 \mathrm{~km}$ (up to $40 \mathrm{~km}$ in pushed configurations), represents a technological challenge.

The detailed analysis of the frequency dependent shielding effect of the beam screen presented in this paper, was motivated by the need to specify the current and voltage stability of the power converters for the HL-LHC IR magnet [12-15]. In general, two regimes can be distinguished for power converter ripples: (i) current control where $f \leq f_{0}$ and the current of the power converter is directly controlled, (ii) voltage control where $f>f_{0}$ and the voltage of the power converter is directly controlled, assuming $f_{0}$ as a parameter of the power converter regulation ranging from few hundreds $\mathrm{mHz}$ to few $\mathrm{Hz}$ for HL-LHC. As it will be shown shortly, the transfer function of the beam screen for the main field component will not introduce any attenuation up to roughly $10 \mathrm{~Hz}$ and therefore the shielding effect of the beam screen only contributes in the voltage control regime. In this case, the following model for the transfer function from the voltage of the power converter to the magnetic field seen by the beam can be assumed:

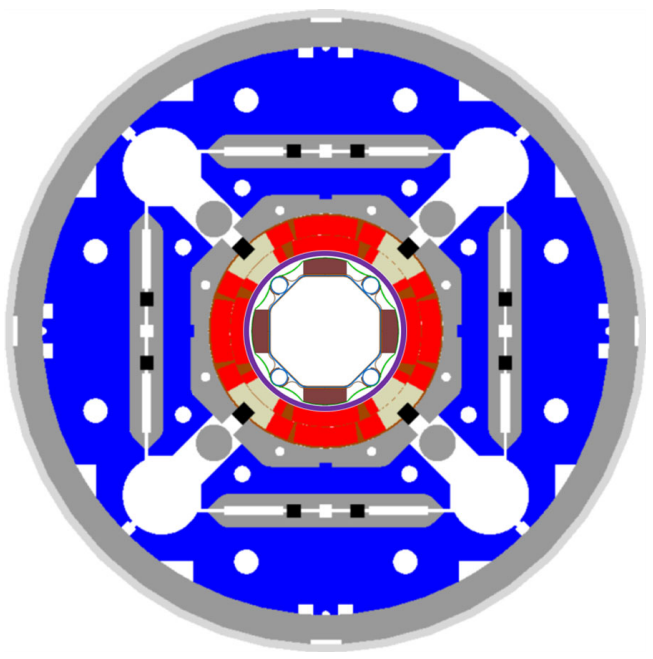

FIG. 2. The new Q1 HL-LHC beam screen inserted in the MQXF magnet. Q2, Q3 magnets have the same cross section but are equipped with a larger beam screen. The red area represents the coils and the beam screen is placed in the aperture surrounded by the cold bore.
$B(f)=T_{\mathrm{B}_{\mathrm{m}} \text { to } \mathrm{B}_{\mathrm{b}}}(f) \times T_{\mathrm{ItoB}_{\mathrm{m}}}(f) \times T_{\mathrm{V} \text { to I }}(f) \times V(f)$

where $f$ is the frequency, $V(f)$ the voltage ripple of the power supply, $T_{\mathrm{V} \text { toI }}(f)$ the admittance of the circuit as seen by the power converter, $T_{\text {Ito } \mathrm{B}_{\mathrm{m}}}(f)$ the transfer function from the input circuit current to the magnetic field, and finally, $T_{\mathrm{B}_{\mathrm{m}} \text { to } \mathrm{B}_{\mathrm{b}}}(f)$ represents the purely magnetic transfer function of the cold bore, absorber, and beam screen (from the $B$ field generated by the magnet to the $B$ field seen by the beam). This paper is devoted to the characterization of

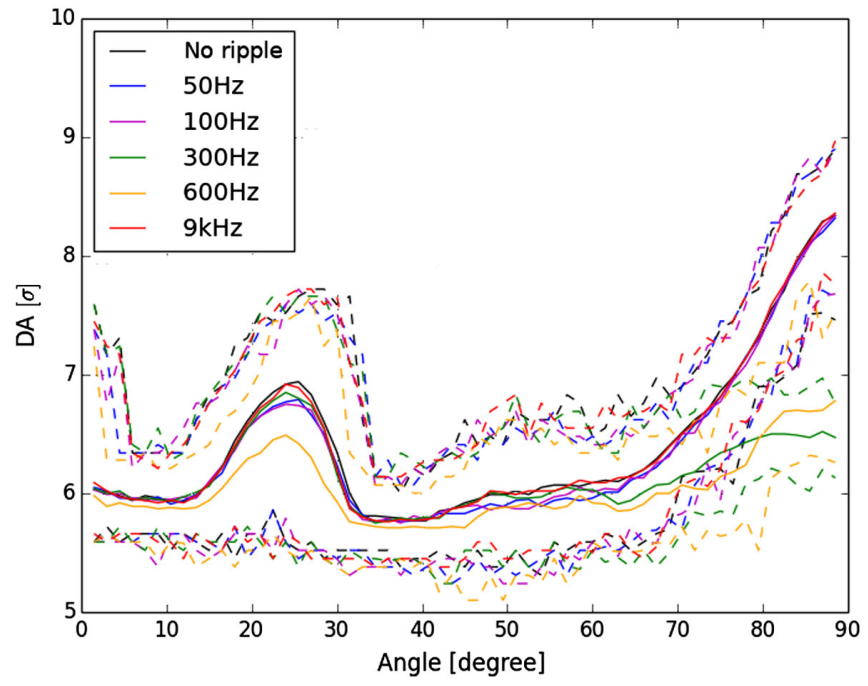

FIG. 3. Impact on the dynamic aperture estimates for HL-LHC of a tune ripple source giving $10^{-4}$ perturbation as a function of ripple frequency. The dynamic aperture is estimated by determining the first initial transverse normalized amplitude for a given azimuthal angle of particles that are lost after $10^{5}$ turns. The calculation is performed on $60 \mathrm{HL}-\mathrm{LHC}$ machine models with a statistical distribution of magnetic field imperfections compatible with the magnetic measurements. The plot shows the average dynamic aperture (continuous lines) and the minimum and maximum dynamic aperture (dashed lines) over the 60 machine models as a function of the azimuthal angle of the initial conditions for different noise frequencies. Tune fluctuations at 300 and $600 \mathrm{~Hz}$ result in dynamic aperture reductions and justify a detailed analysis of how noise sources in this frequency range can propagate inside the vacuum chamber. 
$T_{\mathrm{B}_{\mathrm{m}} \text { to } \mathrm{B}_{\mathrm{b}}}(f)$ and to the best of the authors' knowledge a rigorous characterization of $T_{\mathrm{B}_{\mathrm{m}} \text { to } \mathrm{B}_{\mathrm{b}}}(f)$ has never been presented in literature prior to this publication.

The paper is structured as follows: Sec. II is dedicated to the description of the different HL-LHC beam screens, the FEM modeling and the simulation details. Sec. III presents the post-processing technique used to derive the frequency behavior of the relevant multipole components. In Sec. IV a new analytically approximated formulation is derived. The novelty of this low-frequency formula compared to earlier publications [16-19] is that it also applies to multi-layer conductive shells like those constituting the beam screens foreseen for HL-LHC. Furthermore, $T_{\mathrm{B}_{\mathrm{m}} \text { to } \mathrm{B}_{\mathrm{b}}}(f)$ is properly defined in multipole terms as it is generally done in magnetic measurements for the DC characterization of particle accelerator magnets. Finally, a similar study for the LHC main dipoles and quadrupoles is presented in Sec. V for comparative purposes.

\section{SIMULATION SETUP}

\section{A. Beam screen versions}

The new beam screen is an octagonally-shaped pipe made of high nitrogen-high manganese stainless steel (P506) [20] whose main function is to shield by means of tungsten-based inserts the superconducting magnets from debris coming from the collisions, screening the cold mass from beaminduced heating, and ensuring the vacuum levels required for the beam lifetime. The beam screen is placed within the cold bore made of stainless steel $316 \mathrm{LN}$ of the new superconducting magnets [2,21]. Cross sections of the different configurations are depicted in Fig. 4 and their geometrical dimensions are reported in Table I.

The internal side of the assembly is colaminated with $75 \mu \mathrm{m}$ of high purity copper (RRR 100) to lower the beam impedance. On top of the longitudinal flat surfaces of the beam screen four tungsten-based inserts and four cooling tubes are placed in an alternate way. The inserts are laid on the octagonal pipe to avoid detrimental residual stress during the cool-down [22], while the tubes are laserwelded. The temperature of the beam screen is expected to be between 60 and $80 \mathrm{~K}$ while the cold bore is maintained at $1.9 \mathrm{~K}$. The heat load is therefore intercepted at a higher temperature by the inserts and then transferred to the cooling tubes through small copper links. The evacuation of heat at these temperatures rather than at $1.9 \mathrm{~K}$ lowers dramatically the energy consumption required by the cryogenic system. In addition, the desired vacuum performance of the screen fitted with pumping slots is ensured [23].

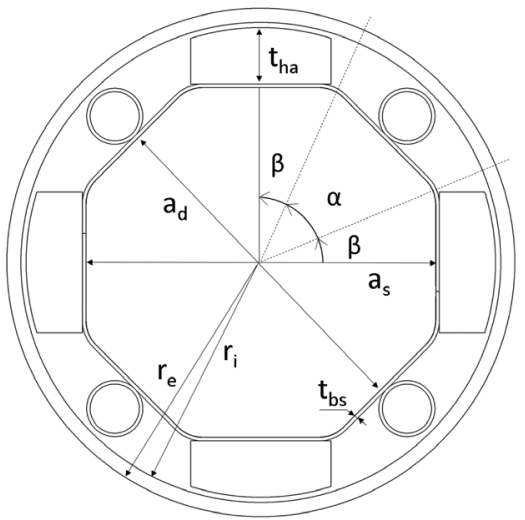

(a) Q1 beam screen cross section.

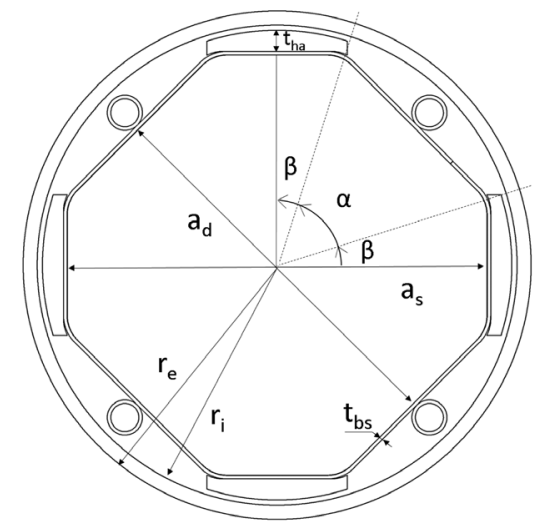

(b) Q2-Q3-D1 beam screen cross section.

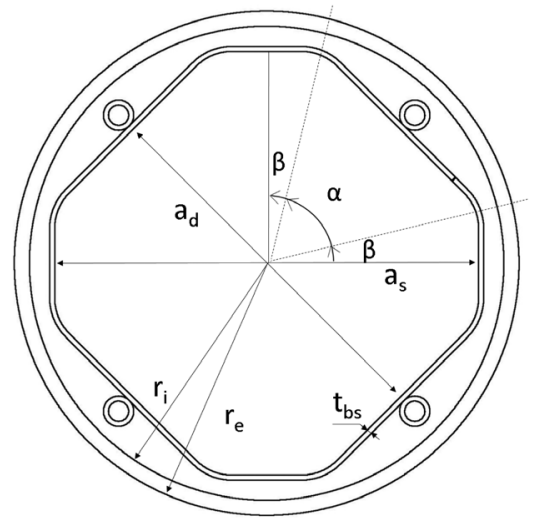

(c) D2 beam screen cross section.

FIG. 4. Different types of beam screens for the IR quadrupoles. 
TABLE I. Characteristic angles [deg] and dimensions [mm] of the beam screens analyzed in this paper, for LHC see Fig. 16.

\begin{tabular}{lccrc}
\hline \hline Symbol & Q1 & Q2-Q3-D1 & D2 & LHC \\
& \multicolumn{1}{c}{ Beam screens angles and dimensions } & \\
$\alpha$ [deg] & 45 & 54.6 & 60 & 52.4 \\
$\beta$ [deg] & 22.5 & 17.7 & 15 & 37.6 \\
$a_{s}[\mathrm{~mm}]$ & 99.7 & 119.7 & 86 & 46.5 \\
$a_{d}[\mathrm{~mm}]$ & 99.7 & 110.7 & 77 & 36.9 \\
$t_{h a}[\mathrm{~mm}]$ & 16 & 6 & $\ldots$ & $\ldots$ \\
$t_{b s}[\mathrm{~mm}]$ & 1 & 1 & 1 & 1 \\
$r_{i}[\mathrm{~mm}]$ & 68.35 & 68.35 & 47 & 25 \\
$r_{e}[\mathrm{~mm}]$ & 72.35 & 72.35 & 50 & 26.5 \\
\hline \hline
\end{tabular}

As discussed in [16,17], the filtering effect of the beam screen depends on the electrical conductivity $\sigma$, where a larger electrical conductivity leads to a stronger filtering effect (lower cutoff frequencies).

As $\sigma$ is inversely proportional to temperature, the worst case scenario within the scope of this work is set to be at $80 \mathrm{~K}$ for the Q1, Q2 (in the following Q2 will be used both for Q2a and Q2b), Q3 and D1 beam screens. Similarly, a $20 \mathrm{~K}$ threshold is assumed for the D2 beam screen that will be kept at the LHC temperature (i.e., $4.5 \mathrm{~K}$ up to $20 \mathrm{~K}$ ).

\section{B. Physics of the model}

The magnetic frequency response of the beam screen is computed through a two-dimensional numerical model implemented in the commercial FEM platform COMSOL Multiphysics [24]. The model hinges on the well known Maxwell's equations, which are considered in their differential form. For clarity, the relevant quantities, symbols, and units are summarized in Table II.

Such a model was originally developed to study the mechanical behavior of the beam screen during a magnet quench [25]. In this model, the magnet coils generating the harmonic field have not been accounted for in the FEM discretization as this would have increased considerably the complexity and the computational load of the simulation. The magnetic field input has been considered more conveniently through the reduced magnet vector potential (RMVP) formulation [26]. This formulation is based on the magnetic vector potential by which $\mathbf{B}$ can be expressed as:

$$
\mathbf{B}=\nabla \times \mathbf{A} .
$$

In turn, the magnetic vector potential is the sum of the reduced potential, $\mathbf{A}_{\text {red }}$, and the known background field, $\mathbf{A}_{\text {ext }}$ :

$$
\mathbf{A}=\mathbf{A}_{\text {red }}+\mathbf{A}_{\text {ext }}
$$

The strategy is to solve only for $\mathbf{A}_{\text {red }}$.
TABLE II. Physical quantities of the constitutive equations governing the beam screen behavior subject to a time-harmonic signal.

\begin{tabular}{lcc}
\hline \hline Quantity & Symbol & SI unit \\
\hline Magnetic flux density & $\mathbf{B}^{\mathrm{a}}$ & $\mathrm{T}$ \\
Magnetic field & $\mathbf{H}$ & $\mathrm{A} \mathrm{m}^{-1}$ \\
Magnetic vector potential & $\mathbf{A}$ & $\mathrm{Wb} \mathrm{m}^{-1}$ \\
Angular frequency & $\omega$ & $\mathrm{rad} \mathrm{s}^{-1}$ \\
Frequency & $f$ & $\mathrm{~Hz}^{-1}$ \\
Electrical conductivity & $\sigma$ & $\mathrm{S} \mathrm{m}^{-1}$ \\
Permittivity & $\epsilon$ & $\mathrm{F} \mathrm{m}^{-1}$ \\
Vacuum permeability & $\mu_{0}$ & $\mathrm{H} \mathrm{m}^{-1}$ \\
Relative permeability & $\mu_{r}$ & \\
External current density & $\mathbf{J}_{e}$ & $\mathrm{~A} \mathrm{~m}^{-2}$ \\
\hline \hline
\end{tabular}

${ }^{\mathrm{a}}$ In the text $\mathbf{B}$ is referred as magnetic field or induction.

Therefore, for a time-harmonic study, the governing equation in the conducting region becomes:

$$
\left(j \omega \sigma-\omega^{2} \epsilon\right) \mathbf{A}+\nabla \times\left(\mu_{0}^{-1} \nabla \times \mathbf{A}\right)=\mathbf{J}_{e} .
$$

where the external current density $\mathbf{J}_{e}$ is 0 for the application treated in this paper.

\section{Boundary conditions}

To account for the magnetic-field interactions, it is necessary to model a medium surrounding the beam screen. Therefore, a cylindrical domain has been created around the assembly. Symmetry conditions are used to lower the computational load of the magnetic simulations. A quarter of the beam screen is sufficient to fully characterize the behavior of the assembly. Figures 5(a) and 5(b) show the boundary conditions for a quarter of the beam screen inserted in a dipole and quadrupole magnet, respectively.

The magnetic insulation boundary condition can be expressed as:

$$
\mathbf{n} \times \mathbf{A}=\mathbf{0},
$$

and the perfect magnetic conductor boundary condition as:

$$
\mathbf{n} \times \mathbf{H}=\mathbf{0} .
$$

The former implies that the magnetic field is zero in the normal direction to the boundary. Therefore, the field can only be tangential. The latter has the opposite effect, i.e., the magnetic field can only be perpendicular to the selected boundary while the tangential component has to be zero. Considering the magnetic field distribution of the dipolar field, see Fig. 5(a), B can be truncated along the y-axis by the magnetic insulation condition and along the $\mathrm{x}$-axis by the perfect magnetic conductor condition. Instead, the quadrupole field can be truncated at $\frac{\pi}{4}$ and $\frac{3}{4} \pi$ angle by the perfect magnetic conductor conditions, see Fig. 5(b). 


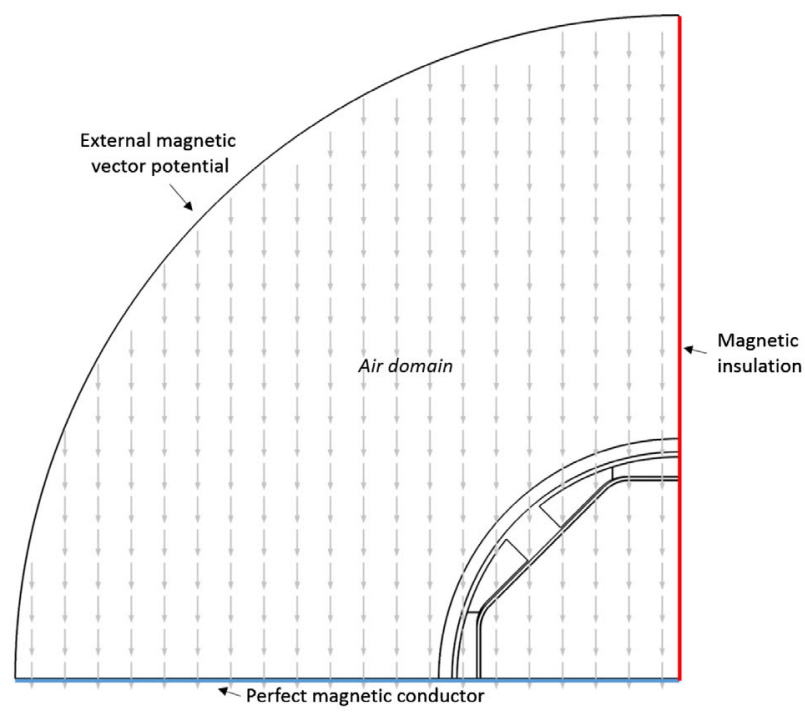

(a) Dipole

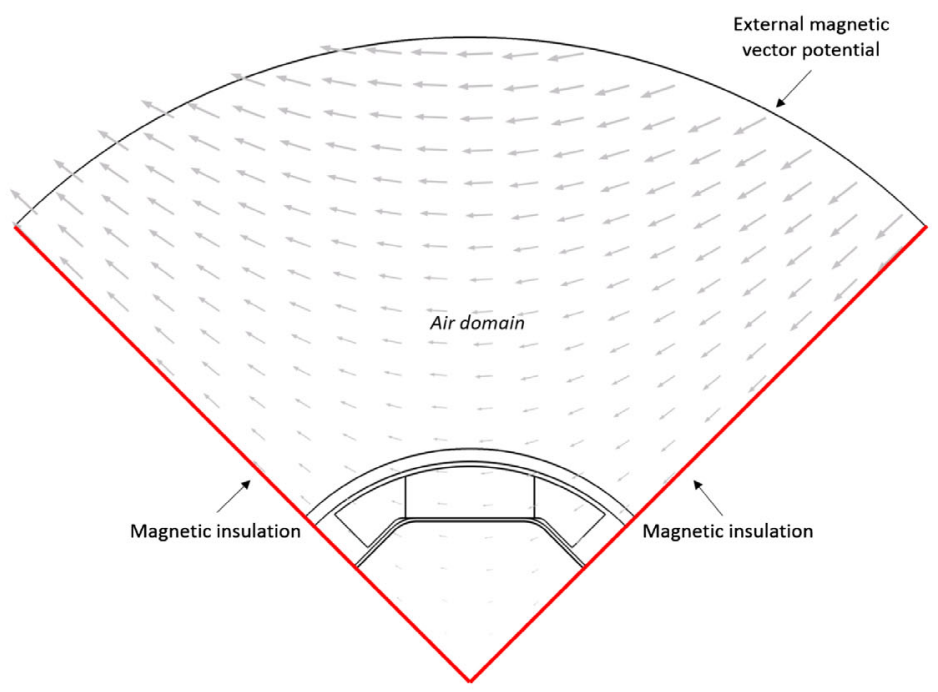

(b) Quadrupole

FIG. 5. Symmetry conditions (in blue and in red) applied on the straight edges to reduce the modelling domain in case of a dipolar (a) and quadrupolar (b) magnetic field. The field lines are shown in gray. For both cases the external vector potential is applied on the round edge.

To use the reduced-field formulation, the total field $\mathbf{A}$ has to be equal to the background field $\mathbf{A}_{b}$ along the outer boundaries of the air domain, see Figs. 5(a) and 5(b). This condition translates into:

$$
\mathbf{n} \times \mathbf{A}=\mathbf{n} \times \mathbf{A}_{b}
$$

\section{Domain discretization}

In electromagnetic problems the mesh discretization depends mainly on the skin depth of the physical domains. The skin depth for a conductive material is:

$$
\delta=\sqrt{\frac{1}{\pi f \mu \sigma}} .
$$

It is recommended that at least two linear elements per skin depth are used to capture the variation of the fields [27]. If the skin depth is much shorter than the geometrical domain it can be replaced by an impedance boundary condition. In the study presented in this paper, the inner copper layer at $20 \mathrm{~K}$ has the highest electrical conductivity, namely $6 \times 10^{9} \mathrm{~S} \mathrm{~m}^{-1}$, and therefore the shortest skin depth amongst all the beam screen materials. At $1000 \mathrm{~Hz}$, the highest frequency analyzed in this study, the skin depth of copper at $20 \mathrm{~K}$ is $206 \mu \mathrm{m}$ which is larger than the thickness of the copper layer itself $(75 \mu \mathrm{m})$. However, considering that the computational time of the simulations is within a few minutes, two quadratic type elements are used to mesh the copper layer, as depicted in Fig. 6.

\section{E. Electrical resistivity of the copper layer}

The results presented in this paper take into account the magnetoresistivity of copper, whose variation of electrical resistivity due to the magnetic field is not negligible in comparison with the other materials of the beam screen. For the quadrupole magnets, the magnetoresistivity is considered by implementing in the material properties of COMSOL a data sheet containing the electrical conductivity as a function of the magnetic field. The LHC beam screen operates in a magnetic field range between 3.69 to $4.65 \mathrm{~T}$, while the HL-LHC version between 7.74 to $8.38 \mathrm{~T}$. A magnet gradient of $200 \mathrm{~T} \mathrm{~m}^{-1}$ has been considered for

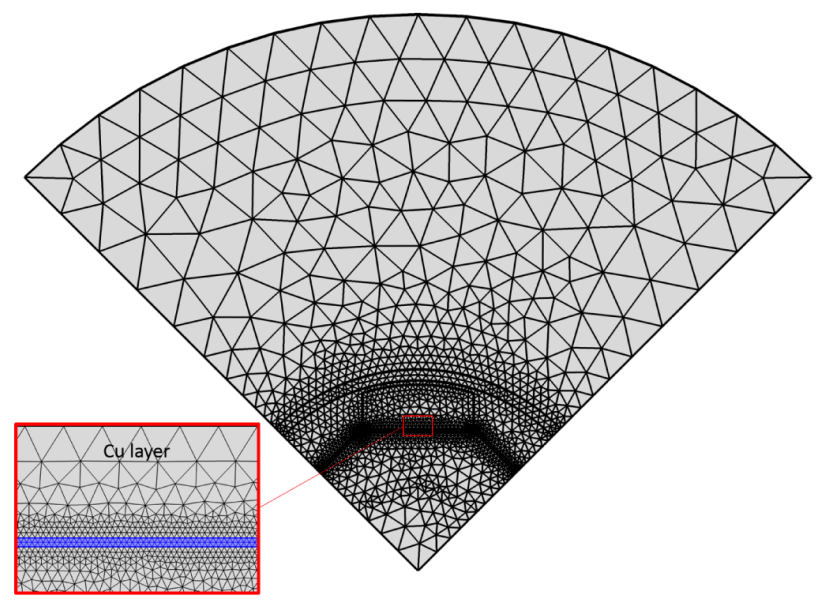

FIG. 6. Domain discretization of the Q1 beam screen including the air box. A magnification of the $\mathrm{Cu}$ layer is shown in the box on the left. 
the LHC main quadrupole (MQ) and of $132.6 \mathrm{~T} \mathrm{~m}^{-1}$ for the quadrupole HL-LHC magnets. The value of electrical resistivity of the dipole magnets has been set constant over the whole beam screen region. The magnetic field considered for the HL-LHC dipole magnets is 5.6 T, while for the LHC is $8 \mathrm{~T}$. In general, the effect of the magnetoresistivity is proportionally more significant at $20 \mathrm{~K}$ than at $80 \mathrm{~K}$ as the intrinsic component of the phonons resistivity is very small at lower temperatures [28]. In fact, for a magnetic field of $5.6 \mathrm{~T}$ the electrical resistivity is $56 \%$ higher at $20 \mathrm{~K}$, while it is only $6 \%$ higher at $80 \mathrm{~K}$. For a magnetic field of $8 \mathrm{~T}$ the electrical resistivity is $65 \%$ higher at $20 \mathrm{~K}$, while it is $9 \%$ higher at $80 \mathrm{~K}$. The assumption adopted for the electrical resistivity of copper is as follows. If $B<0.1 \mathrm{~T}$ the electrical resistivity is based on the Matthiessen's formulation [29]:

$$
\rho=\rho_{0}+\rho_{i}+\rho_{i 0}
$$

where $\rho_{0}$ is the residual resistivity which is determined by the chemical and physical imperfections of the material, $\rho_{i}$ is the intrinsic resistivity affected by the electrons interacting with the phonons, and $\rho_{i 0}$ is a term to account for some observed deviation from experimental data. Considering $\rho(273 K)=15.53 \mathrm{n} \Omega \mathrm{m}$, these quantities read to:

$$
\begin{gathered}
\rho_{0}=\rho(273 K) / R R R \\
\rho_{i}=P_{1} T^{P_{2}} /\left(1+P_{1} P_{3} T^{P_{2}+P_{4}} \exp \left(-\left(P_{5} / T\right)^{P_{6}}\right)\right) \\
\rho_{i 0}=P_{7} \rho_{i} \rho_{0} /\left(\rho_{i}+\rho_{0}\right)
\end{gathered}
$$

The constants are: $P_{1}=1.171 \times 10^{-17}, P_{2}=4.49, P_{3}=$ $3.841 \times 10^{10}, \quad P_{4}=1.14, \quad P_{5}=50, \quad P_{6}=6.428, \quad P_{7}=$ 0.4531. If $B>0.1 \mathrm{~T}$, the electrical resistivity in the direction perpendicular to the magnetic field can be described by the empirical Kohler's rule [29,30]:

$$
\begin{aligned}
\log \left[\rho_{T}(B)-\rho_{T}(0)\right] / \rho_{T}(0) \\
=-2.662+0.3168 \log [B S(T)]+0.6229 \log [B S(T)]^{2} \\
\quad-0.1839 \log [B S(T)]^{3}+0.01827 \log [B S(T)]^{4}
\end{aligned}
$$

where $\rho_{T}(B)$ is the electrical resistivity as a function of the magnetic field at a specific temperature, $\rho_{T}(0)$ is the electrical resistivity with no magnetic field at a specific temperature, $S(T)$ is the ratio between the electrical resistivity at $273 \mathrm{~K}$ and a specific temperature with no magnetic field, i.e., $\rho_{273 K}(0) / \rho_{T}(0)$.

\section{F. Time-domain vs frequency-domain study}

The frequency response of the model has been validated in the time domain for the Q1 beam screen at 10, 100, and $1000 \mathrm{~Hz}$. The comparison has been performed by considering the norm of the magnetic field at $x=0$, $y=49.5 \mathrm{~mm}$. A sinusoidal time-dependent magnetic field with a magnitude of $1 \mathrm{~T}$ has been chosen as the excitation for the time-dependent study. The same magnitude has been used for the quasi-static frequency study. After the transients of the time-domain simulations are vanished, an excellent agreement was found for all frequencies analyzed. Therefore, the study has been efficiently conducted through a stationary problem in the frequency domain with complex-valued solutions. The frequencies, in the range 0.1 to $1000 \mathrm{~Hz}$ with a logarithmically spaced grid of 37 points, have been computed through the direct solver MUMPS [31]. The memory allocation factor used for such solver is 1.2 with the preordering algorithm based on the nested dissection. The relative tolerance for the solver to converge was set to $10^{-3}$. Figure 7 shows the magnetic field map of the D1 beam screen at $278 \mathrm{~Hz}$ using the stationary solver.

\section{G. Contribution of the heat absorber wings}

As shown in Fig. 8 for the Q1 beam screen, the tungstenbased inserts (also called heat absorbers) have some geometrical extensions on both sides known as tungsten wings. As these are not uniformly present along the beam axis, they require a 3D model. However, an effective workaround still allowing the use of a 2D simulation was found in [32]. It consists of defining an equivalent electrical conductivity related to the numbers of wings along the whole length of the heat absorber. For the case study presented in this paper, the geometrical filling ratio of the wings is $20 \%$, therefore, the electrical conductivity was also set to $20 \%$ of the tungsten alloy used in the heat absorbers.

For the D1 and Q2 cases, sharing the same type of beam screen, the percentage difference in terms of magnetic field

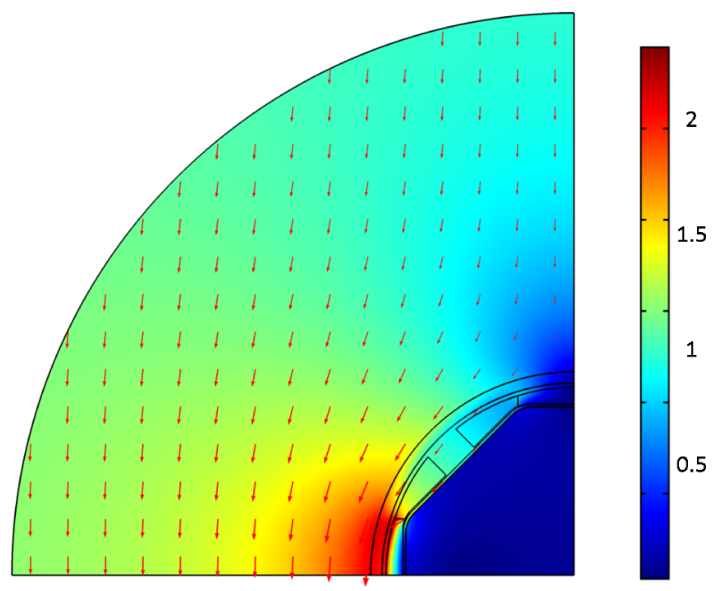

FIG. 7. Magnetic field map around the D1-type beam screen at $278 \mathrm{~Hz}$. The color map, in T, is normalized to the input source. The red vectors represent the magnetic field lines. 


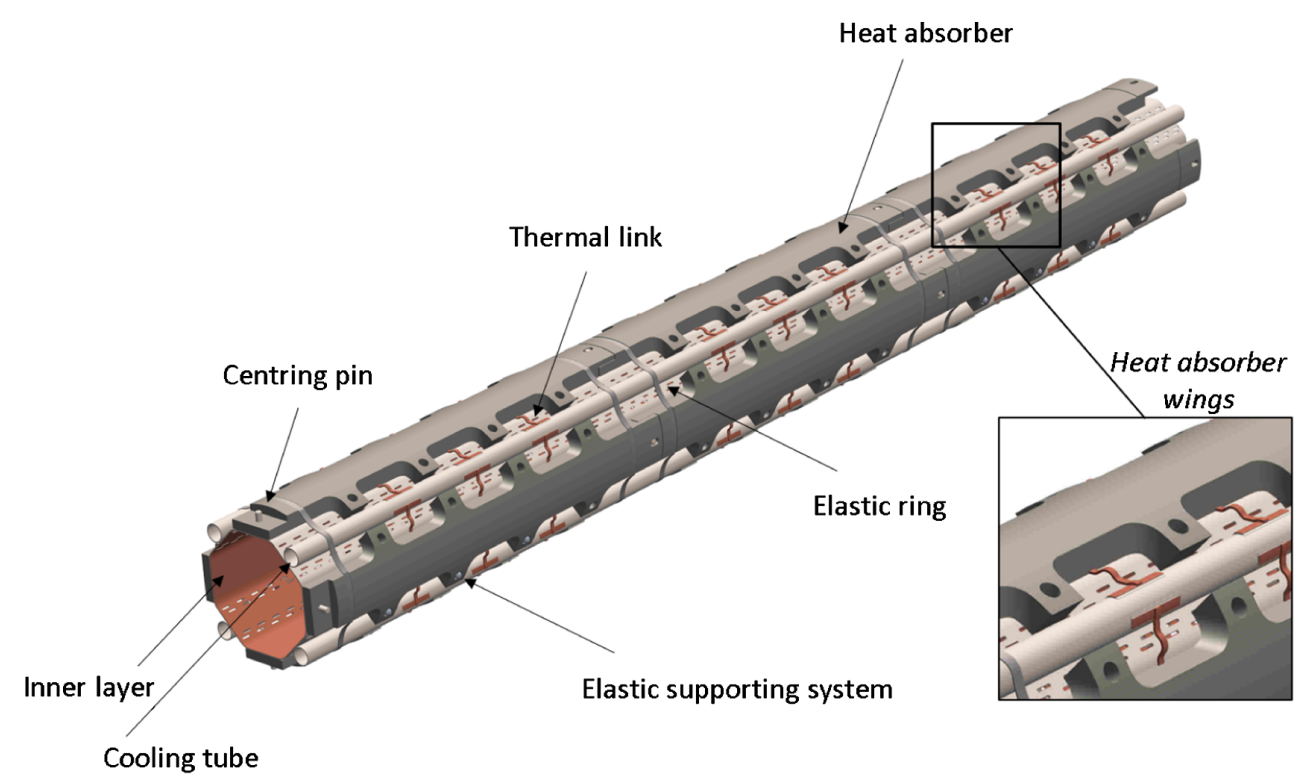

FIG. 8. The new Q1 HL-LHC beam screen.

with and without the tungsten wings is given in Fig. 9. The magnitude of the magnetic field has been compared as a function of the frequency at $x=0, y=0$ of the D1 magnet and at $x=0, y=30 \mathrm{~mm}$ for the Q2 magnet. It turns out that for D1 the shielding effect increases monotonically with frequency. This is due to the extra absorbing material of the wings. For Q2, at the chosen observation point, the shielding effect increases slightly up to $200 \mathrm{~Hz}$ but then decreases from 200 to $1000 \mathrm{~Hz}$. The difference between these two profiles is due to the intrinsic distribution of the dipole and quadrupole magnetic field. However, in the case

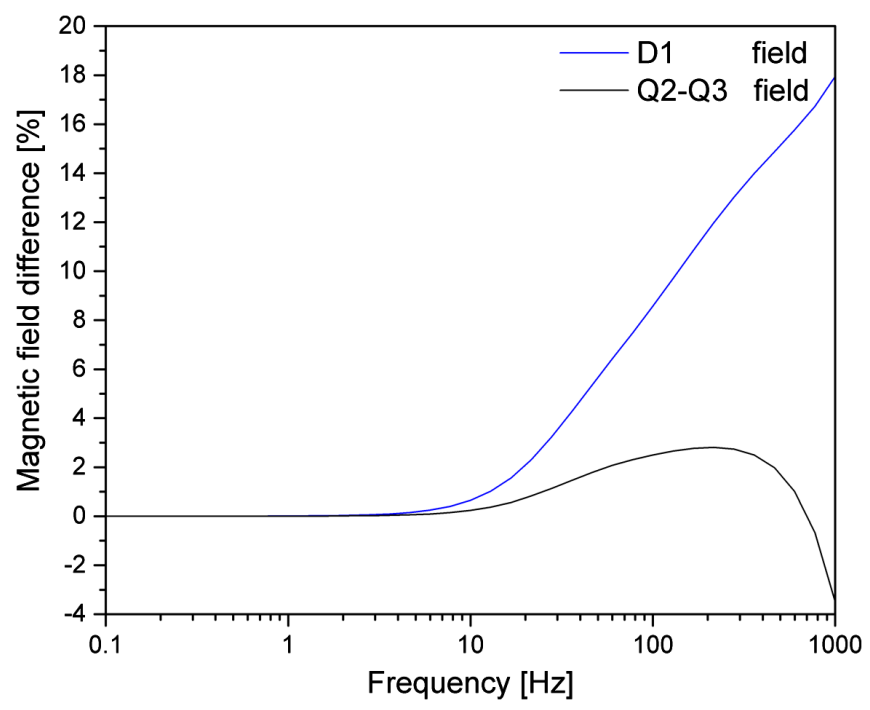

FIG. 9. Percentage difference of the magnetic field between the D1 (in blue) and Q2-Q3 (in black) beam screens with and without wings. Q1 is expected to follow the same behavior of Q2-Q3. of the IT, this difference is deemed negligible for the purpose of this study. The wings of the heat absorbers are therefore not accounted for in the frequency response of the IT beam screens.

\section{H. The inner copper layer}

The nominal thickness of the inner copper layer for both LHC and HL-LHC beam screens is set to $75 \mu \mathrm{m}$ $(-8 \mu \mathrm{m} /+18 \mu \mathrm{m})$. Following colamination, the strip is partially annealed under a protective atmosphere with a partial $\mathrm{O}_{2}$ pressure lower than $10 \mathrm{ppm}$. The annealing process improves the adhesion of the copper layer and ensures an appropriate compromise between mechanical properties (stress relieving) and electrical properties (low temperature surface resistance of the copper) of the finished product. As an indication, partial annealing in a continuous furnace under pure $\mathrm{H}_{2}$ at $920^{\circ} \mathrm{C}$ during 5 minutes has given satisfactory results during the LHC manufacturing runs. However, during the manufacturing process a diffusion mechanism takes place from the stainless steel to the copper layer which affects its purity and, therefore, the electrical conductivity. It turns out that the bottom $25 \mu \mathrm{m}$ of the copper becomes polluted. Some electrical resistance measurements have been performed on the LHC beam screen after manufacturing showing an effective thickness of the copper layer between $50 \mu \mathrm{m}$ and $80 \mu \mathrm{m}$ [30]. The analysis presented in this paper is conservative as it has been performed considering $50 \mu \mathrm{m}$.

\section{POSTPROCESSING}

2D multipole expansions of magnetic fields and the analysis of relative field components is common practice in studying imperfections of accelerator magnets [33]. In this 
analysis, a formalism as well as practical equations are developed in order to define and extract relative field components for time varying fields in the frequency domain based on simulation data.

\section{A. Frequency-dependent multipole analysis}

A 2D translation-invariant, quasi-static magnetic field $\left[B_{x}(x, y, t), B_{y}(x, y, t)\right]$ can be expressed in a source-free region using a $2 \mathrm{D}$ multipole expansion defined by:

$B_{y}(x, y, t)+i B_{x}(x, y, t)=\sum_{n=1}^{\infty}\left[B_{n}(t)+i A_{n}(t)\right] \frac{(x+i y)^{n-1}}{R^{n-1}}$,

where $B_{n}, A_{n}$ are the multipole components featuring an explicit time dependence since $\nabla \cdot \mathbf{B}=0$ and $\nabla \times \mathbf{B}=$ $\mu \epsilon \partial_{t} \mathbf{E} \approx 0$ at low frequency. $R$ is a convenient reference radius (typically $2 / 3$ of the magnet aperture). For brevity, (14) can be rewritten using complex quantities, yielding:

$$
\mathcal{B}(z, t)=\sum_{n=1}^{\infty} \mathcal{C}_{n}(t) \frac{z^{n-1}}{R^{n-1}}
$$

where $\mathcal{B}(z, t)=B_{y}(x, y, t)+i B_{x}(x, y, t), \mathcal{C}_{n}(t)=B_{n}(t)+$ $i A_{n}(t)$ and $z=x+i y$.

If the magnetic field $\mathcal{B}(z, t)$ is calculated in $M$ points $\left\{z_{k}\right\}=z_{0}, \ldots, z_{M-1}$ uniformly placed on a circle of radius $R,(15)$ simplifies to:

$$
\begin{aligned}
\mathcal{B}_{k}(t) & =B_{y}\left(x_{k}, y_{k}, t\right)+i B_{x}\left(x_{k}, y_{k}, t\right) \\
& =\sum_{n=1}^{\infty} \mathcal{C}_{n}(t) e^{i 2 \pi k(n-1) / M},
\end{aligned}
$$

where $z_{k}=x_{k}+i y_{k}=R \cdot e^{i 2 \pi k / M}$ for $k=0, \ldots,(M-1)$.

Under the assumption that multipole components of order larger than $M / 2$ can be neglected ( $M=64$ has been used in the following analysis to avoid any risk of aliasing effects) and $\mathrm{M}$ is a multiple of 2 , the multipole components of the order up to $M / 2$ can be efficiently extracted from simulations by applying a fast Fourier transform (FFT) on the complex signal $\left\{\mathcal{B}_{0}(t), \ldots, \mathcal{B}_{M-1}(t)\right\}$ because:

$$
\mathcal{C}_{n}(t) \simeq \frac{1}{M} \sum_{k=0}^{M-1} \mathcal{B}_{k}(t) e^{-i 2 \pi k(n-1) / M}
$$

If the field is periodic with a frequency $f$ also the multipole coefficients are periodic. The multipole components for each frequency $f$ can be extracted from the fields calculated by a frequency domain simulations. The fields are normally given in the terms of in-phase (I) and quadrature $(\mathrm{Q})$ components which are defined by:

$$
\begin{aligned}
& B_{y}(x, y, t)=\Re\left\{\left(B_{y}^{\mathrm{I}}(x, y, f)+j B_{y}^{\mathrm{Q}}(x, y, f)\right) e^{j 2 \pi f t}\right\}, \\
& B_{x}(x, y, t)=\Re\left\{\left(B_{x}^{\mathrm{I}}(x, y, f)+j B_{x}^{\mathrm{Q}}(x, y, f)\right) e^{j 2 \pi f t}\right\},
\end{aligned}
$$

in which the complex variable $j$ is used as the imaginary unit of the complex plane related to the frequency domain to distinguish it from $i$, related to complex plane associated to the $2 \mathrm{D}$ fields, and $\Re$ denotes the real part of the complex fields.

Each multipole component will have an amplitude and a phase, therefore each multipole could be denoted by an I and Q component $\left(B_{n}^{\mathrm{I}}(f), B_{n}^{\mathrm{Q}}(f), A_{n}^{\mathrm{I}}(f), A_{n}^{\mathrm{Q}}(f)\right)$ or amplitude and phase components in a complex number:

$$
\begin{aligned}
& \bar{B}_{n}(f)=B_{n}^{\mathrm{I}}(f)+j B_{n}^{\mathrm{Q}}(f) \\
& \bar{A}_{n}(f)=A_{n}^{\mathrm{I}}(f)+j A_{n}^{\mathrm{Q}}(f) .
\end{aligned}
$$

Since the time dependence is separable from the spatial dependence, the multipole analysis can be carried out separately for I and Q components of the field, resulting in I and Q components of each multipole coefficients as defined below:

$$
\begin{aligned}
& B_{y}^{\mathrm{I}}(x, y, f)+i B_{x}^{\mathrm{I}}(x, y, f) \\
& \quad=\sum_{n=1}^{\infty}\left[B_{n}^{\mathrm{I}}(f)+i A_{n}^{\mathrm{I}}(f)\right] \frac{(x+i y)^{n-1}}{R^{n-1}}, \\
& B_{y}^{\mathrm{Q}}(x, y, f)+i B_{x}^{\mathrm{Q}}(x, y, f) \\
& \quad=\sum_{n=1}^{\infty}\left[B_{n}^{\mathrm{Q}}(f)+i A_{n}^{\mathrm{Q}}(f)\right] \frac{(x+i y)^{n-1}}{R^{n-1}},
\end{aligned}
$$

or using complex variables:

$$
\begin{aligned}
& \mathcal{B}^{\mathrm{I}}(z, f)=\sum_{n=1}^{\infty} \mathcal{C}_{n}^{\mathrm{I}}(f) \frac{z^{n-1}}{R^{n-1}}, \\
& \mathcal{B}^{\mathrm{Q}}(z, f)=\sum_{n=1}^{\infty} \mathcal{C}_{n}^{\mathrm{Q}}(f) \frac{z^{n-1}}{R^{n-1}} .
\end{aligned}
$$

When analyzing the field imperfections of a magnet, it is furthermore convenient to express higher order multipoles relative to the main multipole leading to:

$$
\begin{aligned}
& \bar{B}_{n}(f)=B_{n}^{\mathrm{I}}(f)+j B_{n}^{\mathrm{Q}}(f) \\
& \bar{b}_{n}(f)=\bar{B}_{n}(f) / \bar{B}_{N}(0) \\
& \bar{A}_{n}(f)=A_{n}^{\mathrm{I}}(f)+j A_{n}^{\mathrm{Q}}(f) \\
& \bar{a}_{n}(f)=\bar{A}_{n}(f) / \bar{B}_{N}(0)
\end{aligned}
$$



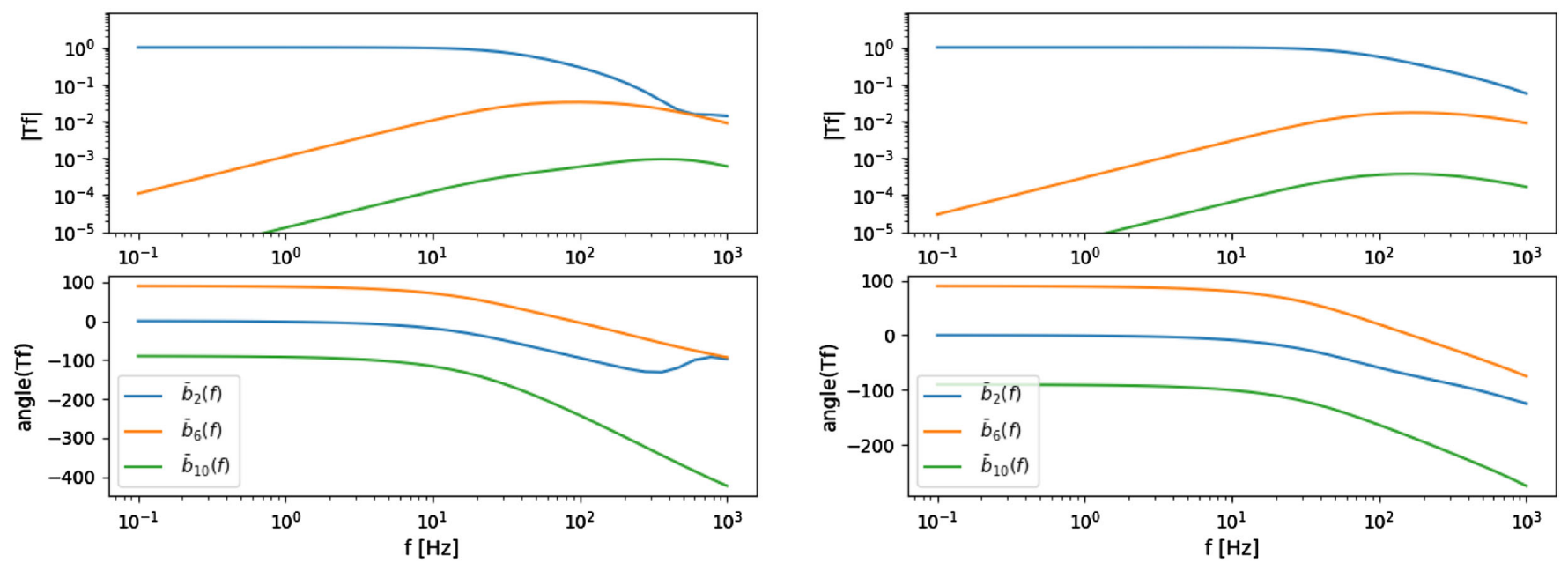

(a) AC multipole analysis for Q1.

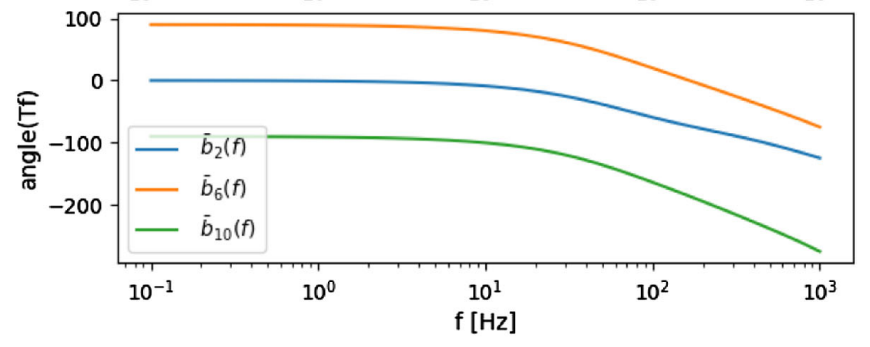

(b) AC multipole analysis for Q2-Q3.

FIG. 10. AC multipole analysis for the beam screens of the HL-LHC triplet quadrupoles at $80 \mathrm{~K}$.

where $\bar{B}_{N}(0)$ is the main DC field component (e.g., $\bar{B}_{1}(0)$ for a dipole, $\bar{B}_{2}(0)$ for a quadrupole), $\bar{B}_{n}(f), \bar{A}_{n}(f)$ are the absolute AC multipole field components, and $\bar{b}_{n}(f)$ and $\bar{a}_{n}(f)$ are the relative AC multipole field components.

\section{B. Relative multipole frequency responses}

Figures 10 and 11 (and also Fig. 14 for LHC) show the amplitude and phase of the AC relative field components resulting from the beam screen of each IR magnets. For the symmetry of field and geometry $\bar{b}_{1}(f), \bar{b}_{3}(f), \bar{b}_{5}(f)$ have the largest amplitudes for dipoles and $\bar{b}_{2}(f), \bar{b}_{6}(f), \bar{b}_{10}(f)$ have the largest amplitudes for quadrupoles. Other components have negligible amplitudes or appear as numerical noise in the processed data. The analysis has been validated by computing the multipole components for different

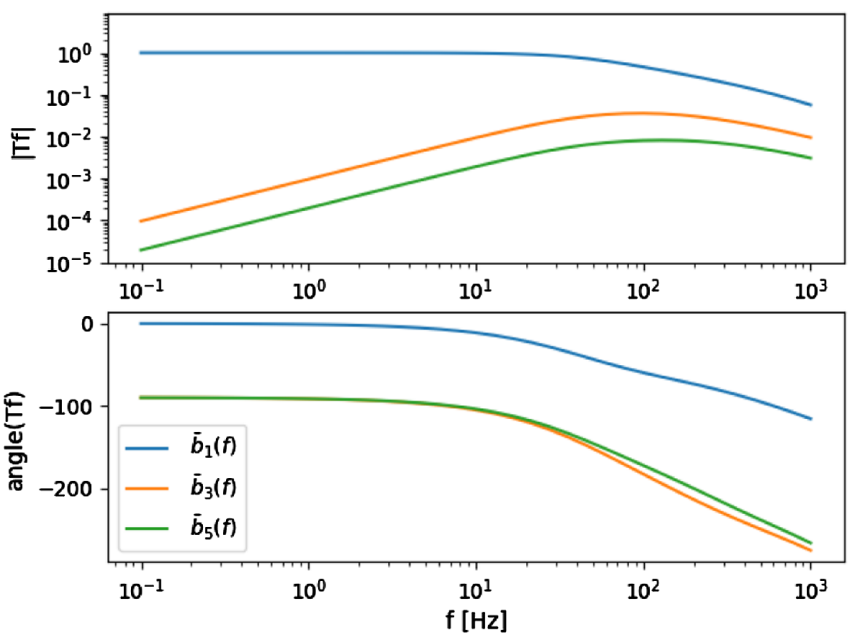

(a) AC multipole analysis for D1 at $80 \mathrm{~K}$. reference radii and verifying how the ideal multipole scaling with radius hold between the different curves. Main field components scale exactly to numerical precision as the reference radius for low frequency with small deviations of the order of $10^{-3}$ at high frequency. Higher orders are less precise but still acceptable in the whole spectrum. The ratio between different reference radii calculated from the ratio of the multipole components is always within $10^{-3}$ of the expected value. The shielding effect of the beam screen and the cold bore is clearly visible on the main field component with different cutoff frequencies (see Table IV). Higher order multipole amplitudes in general increase initially with frequency as the shielding is not homogeneous in the region due to the geometry of the conductors that carry the eddy currents. They then decrease at high frequencies as the cold bore (normally contributing

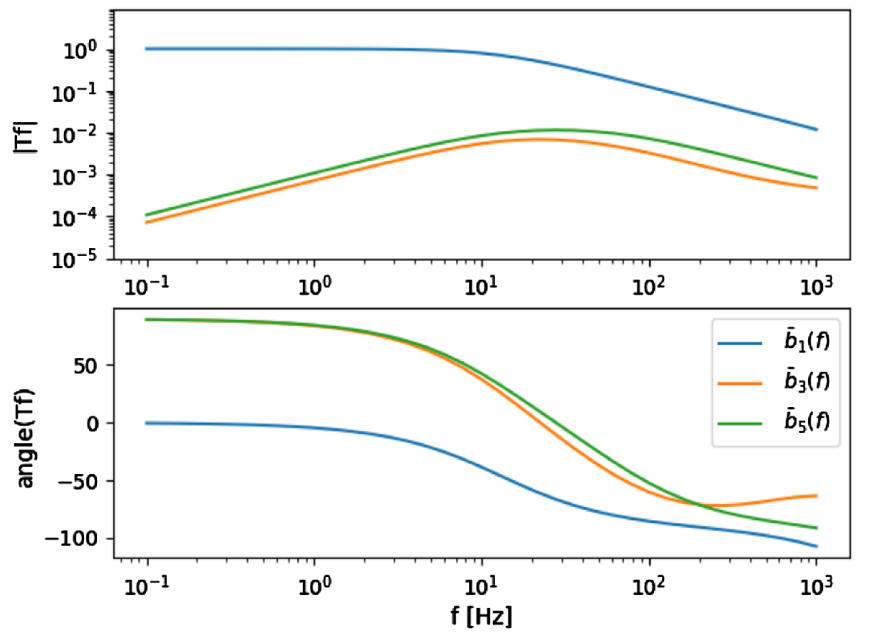

(b) AC multipole analysis for D2 at $20 \mathrm{~K}$.

FIG. 11. AC multipole analysis for the beam screens of the HL-LHC separation-recombination dipoles. 
less to the shielding) reduces the field that generates the eddy currents in the beam screen.

\section{APPROXIMATED ANALYTICAL FORMULATION}

A simplified analytical derivation was first presented in [16] for the case of a cylindrical, infinitely thin (and infinitely long) conductive shell. That formulation, which also took into account the magnet's multipole order, aims at evaluating $T_{\mathrm{B}_{\mathrm{m}} \text { toB }}(f)$ from a beam pipe (as well as obtaining an equivalent circuit model [16]) and it has been used to estimate the potential effects of the HL-LHC beam screen [15]. The expression presented for the cutoff frequency was then generalized, for dipolar field, to an arbitrary cross section in [18], where also elliptical and rectangular shapes are considered and [17] assuming a thin (but not infinitely thin) conductive shell together with experimental results for its validation. A formula for the transfer function of thick round beam pipe was also presented in [19]. In this section, this formulations will be further extended to a more complex shape of the beam screen and validated with the simulation results obtained taking the complex geometry and material layering into account.

\section{A. Low-frequency transfer function derivation}

An analytical derivation, different from those found in $[16,17]$ is presented here for the case of dipole field in order to better highlight its validity range. An infinitely long cylindrical thin conductive shield with a $\mathbf{B}$ field orthogonal to its axis is considered. A closed-form analytical solution exists for the shielding efficiency $(S E)$ of such a simplified structure, as reported in [34]. However, for the scope of this low-frequency characterization and assuming that the shield thickness $\Delta$ is small compared to the average radius $\bar{\rho}_{0}$ of the structure (averaged between inner and outer radii) the following expression holds for $|\gamma \Delta| \ll 1$ [34]:

$$
S E \approx\left|1+\frac{1}{2} \frac{\left(\mu_{r}-1\right)^{2}}{\mu_{r} \bar{\rho}_{0}} \Delta+\frac{\bar{\rho}_{0}}{2 \mu_{r}} \Delta \gamma^{2}\right|
$$

where $\gamma \approx \sqrt{j 2 \pi f \mu_{0} \mu_{r} \sigma}=(1+j) / \delta$. For the structure under analysis clearly $\mu_{r} \approx 1$, so the shielding efficiency can be further simplified to:

$$
S E \approx\left|1+j \pi f \mu_{0} \bar{\rho}_{0} \sigma \Delta\right| .
$$

As the attenuation introduced by the beam screen is simply the inverse of the shielding efficiency, the magnitude of the frequency response is then given by:

$$
|T(f)| \approx\left|\frac{1}{1+j \pi f \mu_{0} \overline{\rho_{0}} \sigma \Delta}\right|=\left|\frac{1}{1+j f / f_{\text {cut }}}\right| .
$$

$T(f)$ will be used in the following as a shorthand for $T_{\mathrm{B}_{\mathrm{m}}}$ to $\mathrm{B}_{\mathrm{b}}(f)$. (30) represents the amplitude of the frequency response of a single pole low-pass filter with a cutoff frequency:

$$
f_{\text {cut }}=\frac{1}{\mu_{0} \pi \bar{\rho}_{0} \Delta \sigma} .
$$

This equation is identical to that presented in [17] for a dipole.

\section{B. Generalization of the approximated formula}

According to [16], as also detailed in Appendix, the cutoff frequency for an $n$th order multipole field is

$$
f_{0}=n f_{\text {cut }}
$$

Only the cold bore can be approximated as an infinitely long cylinder for which (32) immediately applies. The expression for the cutoff frequency in (31), however, can also be rewritten in terms of the annulus area occupied by the shielding material with conductivity $\sigma$ and generalized as:

$$
f_{0}=\frac{2 n}{\mu_{0} A \sigma}
$$

where $A=2 \pi \bar{\rho}_{0} \Delta$ is the cross-section area of the shield. On the other hand, the eddy currents for a $n$th order multipole (for a purely cylindrical conductive shell) have a magnitude proportional to $|\cos n \theta|$ as shown in [16] (and in Appendix). The $A \sigma$ product can be calculated summing up according to the magnitude of the eddy currents as follows:

$$
A \sigma=\int_{0}^{2 \pi} \bar{\rho}_{0} \Delta \sigma k|\cos n \theta| d \theta .
$$

It is straightforward to determine that the proportionality constant $k$ must be equal to $\frac{\pi}{2}$ in order, for (34) to be equal to $2 \pi \bar{\rho}_{0} \Delta \sigma$.

For a noncylindrical conductive shell the magnitude of the eddy currents is no longer strictly proportional to $|\cos n \theta|$; furthermore even for a cylindrical shell whose conductivity is not uniform such proportionality alone is not sufficient to describe the magnitude of the eddy currents. This is the case, as an example, of the magnetoresistive effect in the case of magnet of order $2 n$ when $n>1$ and it will be briefly addressed in VA. The derivation of the approximated formula will be based on the assumption of uniform conductivity. In the following we also assume a closed geometry with an arbitrary number of layers, such that the unwanted higher order components of the magnetic field are, in the worst case, at least one order of magnitude smaller than the $n$th component 
(Figs. 10 and 11) in the range of frequency of interest. Within this approximation, the induced magnetic vector potential is approximately proportional, and opposite, to the source. Therefore the corresponding current density of the induced potential has, approximately, the same azimuthal dependency of the source which, in absolute value, is proportional to $|\cos n \theta|$. In other words the idea is to find a cylindrical approximation for a geometrically complex multi-layer beam screen for which, however, all the quantities in the integral (34) depend on $\theta$. The error introduced by these approximations needs to be verified for specific case with detailed simulations as done in the following sections.

\section{HL-LHC beam screens}

By considering the octagonal shape of the HL-LHC beam screens and the different materials that constitute it, a weighted average area-conductivity product of the cross section of the structure can therefore be evaluated as follows: (i) using an equivalent circular approximation with radius $\bar{\rho}_{\phi}$ for each side of the octagon and then correct for the area with a factor $F_{\phi}=\sin (\phi / 2) / \phi / 2$ (ratio between the actual length of the side and the length of the arc; this applies for both $\phi=\alpha$ and $\phi=2 \beta$ in Fig. 4, only for $\beta$ in Fig. 16); (ii) for each sector forming the $(\Delta \sigma)_{\phi}$ product as $\sum_{j_{\phi}} \Delta_{j_{\phi}} \sigma_{j_{\phi}}$ where $j_{\phi}$ represents the different material layers within the angle $\phi$; (iii) integrating $(\Delta \sigma)_{\phi}$ over $2 \pi$, sector by sector, weighing with $\frac{\pi}{2}|\cos n \theta|$; (iv) adding the conductivity product of the cold bore to the estimated one $\widehat{A \sigma}=2 \pi \bar{\rho}_{C B} \Delta_{C B} \sigma_{316 L N}+\widehat{A \sigma}_{B S}$ (the cold bore area-conductivity product representing a small fraction of the overall one). By symmetry of the structure the general formula for the equivalent area-conductivity product can be easily expressed as:

$\widehat{A \sigma}_{B S}=4 \frac{\pi}{2}\left[\left(c_{\beta}^{(n)}+c_{\bar{\beta}}^{(n)}\right) F_{2 \beta} \bar{\rho}_{\beta}(\Delta \sigma)_{\beta}+c_{\alpha}^{(n)} F_{\alpha} \bar{\rho}_{\alpha}(\Delta \sigma)_{\alpha}\right]$.

The constants introduced are

$$
\begin{aligned}
& c_{\beta}^{(n)}=\int_{0}^{\beta}|\cos (n \theta)| d \theta ; \\
& c_{\alpha}^{(n)}=\int_{\beta}^{\beta+\alpha}|\cos (n \theta)| d \theta ; \\
& c_{\bar{\beta}}^{(n)}=\int_{\beta+\alpha=\frac{\pi}{2}-\beta}^{\frac{\pi}{2}}|\cos (n \theta)| d \theta .
\end{aligned}
$$

Since the area-conductivity product of the cold bore is much smaller than the one of the beam screen, its cutoff frequency alone is much larger; one can alternatively think that higher order multipole components of the field would experience the filtering due to the cold bore whereas the main component would experience mostly the attenuation due to the beam screen.

\section{Numerical validation}

It must be pointed out here that the approximated formula in (28) is valid for $|\gamma \Delta| \ll 1$ which is a safe assumption for the $-3 \mathrm{~dB}$ point. In order to also guarantee accuracy for higher frequencies, a more general formula would be needed, but this outgoes the scope of this paper. It can be stated, however, that as (28) is in turn derived from first order Taylor series of terms in sinh $(\gamma \Delta)$ and $\cosh (\gamma \Delta)$, the filtering effect at higher frequencies is going to be stronger than what can be calculated by means of (30); this holds true for magnets of any order.

From (35) and the constants in (36) the cutoff frequency can be estimated both for dipole and quadrupole configurations as:

$$
\hat{f}_{0}=\frac{2 n}{\mu_{0} \hat{A \sigma}} .
$$

This equivalent cutoff frequency can then be calculated for all configurations of the beam screen in terms of the dimensions reported in Table I and conductivities listed in Table III $[29,35,36]$. The results are summarized in Table IV together with a single pole fit [i.e., with the model described by Eq. (30)] of the relative multipole frequency response obtained in simulations. All the values reported in the table have been derived by means of the nonlinear least-square fit Trust-Region algorithm (disabling any robust option) as available in Curve Fitting Tool of MATLAB and all frequencies have been weighted equally. For comparison, the simulation results together with the best fit and the analytical formulas are shown in Figs. 12 and 13.

In Fig. 13(a) it can be observed that the simulated frequency response, in blue, drops significantly faster after $100 \mathrm{~Hz}$. This is probably due to the $16 \mathrm{~mm}$ thick

TABLE III. Conductivities of materials used for the evaluation of $\hat{f}_{0}$ at their relative temperatures.

\begin{tabular}{lccc}
\hline \hline Material & $\begin{array}{c}\text { Conductivity } \\
{[\mathrm{S} / \mathrm{m}]}\end{array}$ & $\begin{array}{c}\text { Temperature } \\
{[\mathrm{K}]}\end{array}$ & $\begin{array}{c}\text { Magnetic } \\
\text { induction }[\mathrm{T}]\end{array}$ \\
\hline$\sigma_{316 \mathrm{LN}}$ & $1.81 \times 10^{6}$ & 1.9 & $\ldots$ \\
$\sigma_{\mathrm{P} 506}$ & $1.81 \times 10^{6}$ & 20 & $\ldots$ \\
$\sigma_{\mathrm{P} 506}$ & $1.71 \times 10^{6}$ & 80 & $\ldots$ \\
$\sigma_{\mathrm{W}}$ & $2.25 \times 10^{7}$ & 80 & $\ldots$ \\
$\sigma_{\mathrm{Cu}}$ & $4.17 \times 10^{8}$ & 80 & 5.6 \\
$\sigma_{\mathrm{Cu}}$ & $2.64 \times 10^{9}$ & 20 & 5.6 \\
$\sigma_{\mathrm{Cu}}$ & $4.05 \times 10^{8}$ & 80 & 8 \\
$\sigma_{\mathrm{Cu}}$ & $2.09 \times 10^{9}$ & 20 & 8 (LHC Dipole) \\
$\sigma_{\mathrm{Cu}}$ & $2.95 \times 10^{9}$ & 20 & 4.65 (LHC Quadrupole) \\
$\sigma_{\mathrm{Cu}}$ & $3.33 \times 10^{9}$ & 20 & 3.69 (LHC Quadrupole) \\
\hline \hline
\end{tabular}


TABLE IV. Estimation of $\hat{f}_{0}$ for the different cases studied and comparison with numerical fit of the simulated data (assuming single pole behavior). (*): the fit with robust options activated gives better estimations between $59.4 \mathrm{~Hz}$ and $59.6 \mathrm{~Hz}$ and hence an error not greater than $10.7 \%$. For the sake of uniformity though the fitted cutoff frequencies reported here have been calculated with the same algorithm and settings.

\begin{tabular}{lccc}
\hline \hline Magnet & $\hat{f}_{0}$ & Fit & Error \\
\hline D1 $(*)$ & $53.3 \mathrm{~Hz}$ & $61.6 \mathrm{~Hz}$ & $-13.5 \%$ \\
D2 & $46.3 \mathrm{~Hz}$ & $44.5 \mathrm{~Hz}$ & $+4.0 \%$ \\
Q1 & $31.2 \mathrm{~Hz}$ & $31.8 \mathrm{~Hz}$ & $-1.9 \%$ \\
Q2-Q3 & $75.8 \mathrm{~Hz}$ & $71.9 \mathrm{~Hz}$ & $+5.4 \%$ \\
\hline \hline
\end{tabular}

tungsten-based heat absorbers on the $\rho_{\beta}$ sides which conflicts with the assumption $|\gamma \Delta| \ll 1$.

\section{LHC MAIN MAGNETS}

For comparison, the simulation results of the LHC main dipoles and quadrupoles are presented in this section. The obtained results are also deemed interesting in order to better understand in which frequency range the ripple of the currently installed LHC power converters can impact the stability of the beam. The LHC beam screen is illustrated in Fig. 16. For this geometry the formula in (35) simplifies to:
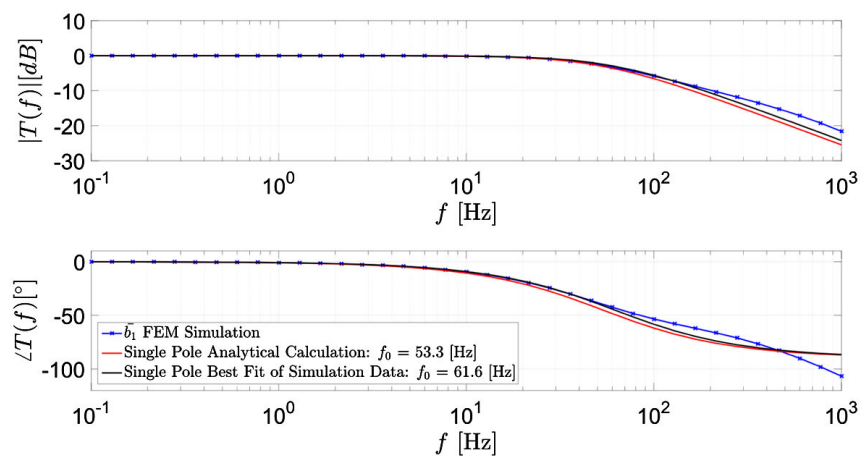

(a) D1 at $80 \mathrm{~K}$
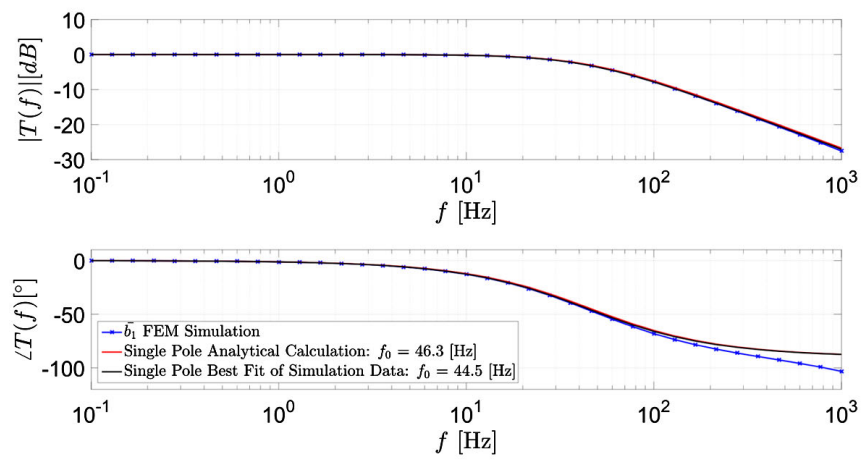

(b) D2 at $20 \mathrm{~K}$

FIG. 12. Frequency response of the shielding effect inside the HL-LHC separation-recombination dipoles.
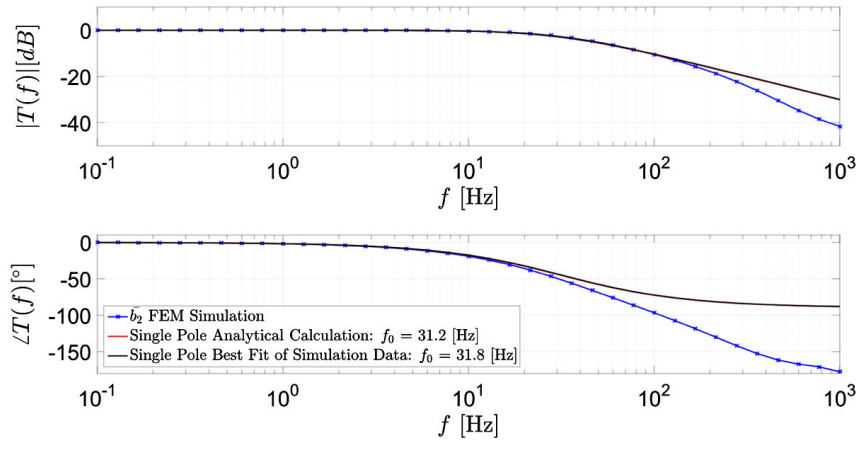

(a) Q1 at $80 \mathrm{~K}$
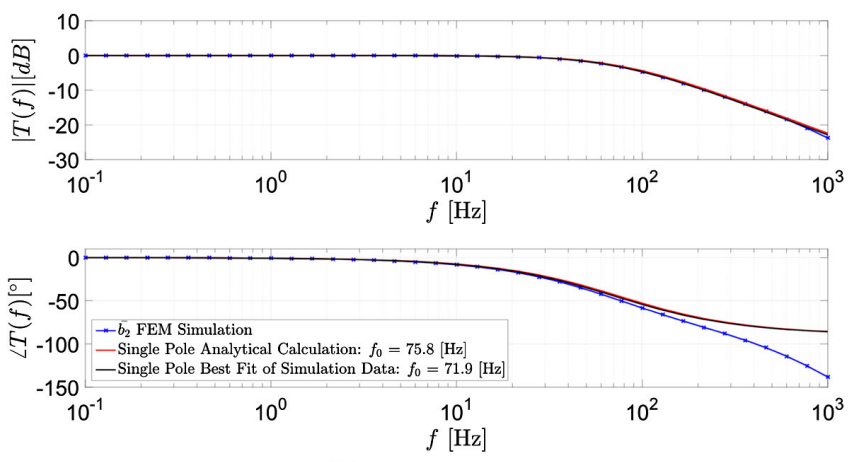

(b) Q2-Q3 at $80 \mathrm{~K}$

FIG. 13. Frequency response of the shielding effect inside the HL-LHC quadrupoles.

$$
\hat{A \sigma_{B S}}=4 \frac{\pi}{2}\left[c_{\bar{\beta}}^{(n)} F_{2 \beta} \bar{\rho}_{\beta}(\Delta \sigma)_{\beta}+c_{\alpha}^{(n)} \bar{\rho}_{\alpha}(\Delta \sigma)_{\alpha}\right],
$$

as the beam screen is actually circular within the angle $\alpha$.

The results for the cutoff frequency are summarized in Table $\mathrm{V}$ based on the AC multipole analysis depicted in Fig. 14 and the simulations shown in Fig. 15. For the case of LHC quadrupoles, the part of the beam screen within the angle $\alpha$ sees an approximately constant $\mathbf{B}$ field of $4.65 \mathrm{~T}$, whereas the part within the angle $\beta$ sees, in the counterclockwise sense, a decreasing field whose minimum is of $3.69 \mathrm{~T}$ when $\beta=\pi / 2$. Here it is simply assumed that the conductivity is constant and equal to the one within angle $\alpha$ (worst case as it represent the lowest conductivity); this means that the calculated cutoff frequency is overestimated. As it can be seen from Table $\mathrm{V}$, the accuracy of $\hat{f}_{0}$ is very good, even for the quadrupoles where the conductivity cannot be assumed uniform.

TABLE V. Estimation of $\hat{f}_{0}$ for the LHC main dipoles and quadrupoles and comparison with numerical fit of the simulated data (assuming single pole behavior).

\begin{tabular}{lccc}
\hline \hline Magnet & $\hat{f_{0}}$ & Fit & Error \\
\hline Dipole & $99 \mathrm{~Hz}$ & $106 \mathrm{~Hz}$ & $-6.6 \%$ \\
Quadrupole & $145 \mathrm{~Hz}$ & $135 \mathrm{~Hz}$ & $+7.4 \%$ \\
\hline \hline
\end{tabular}




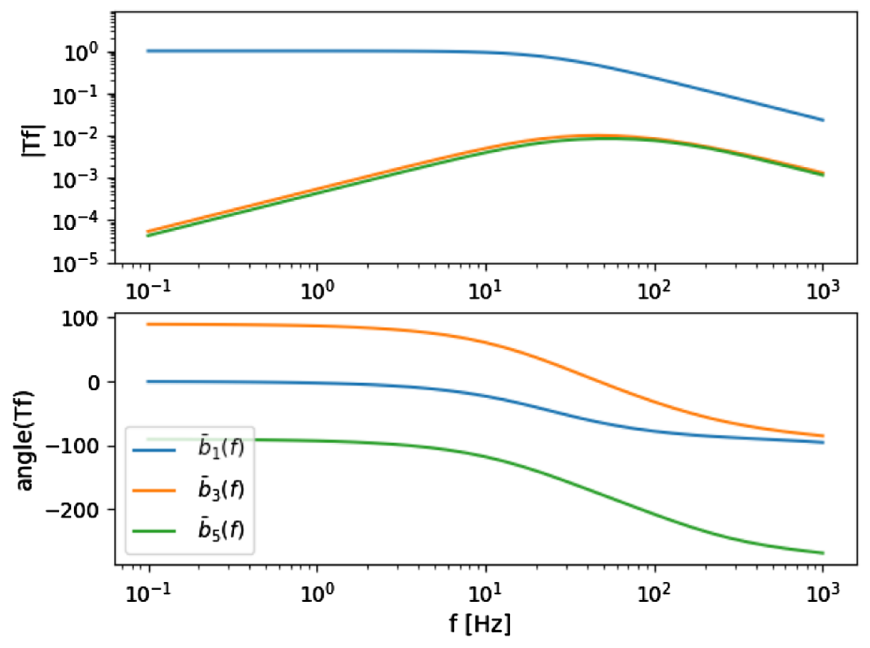

(a) LHC Main Bending at $20 \mathrm{~K}$.

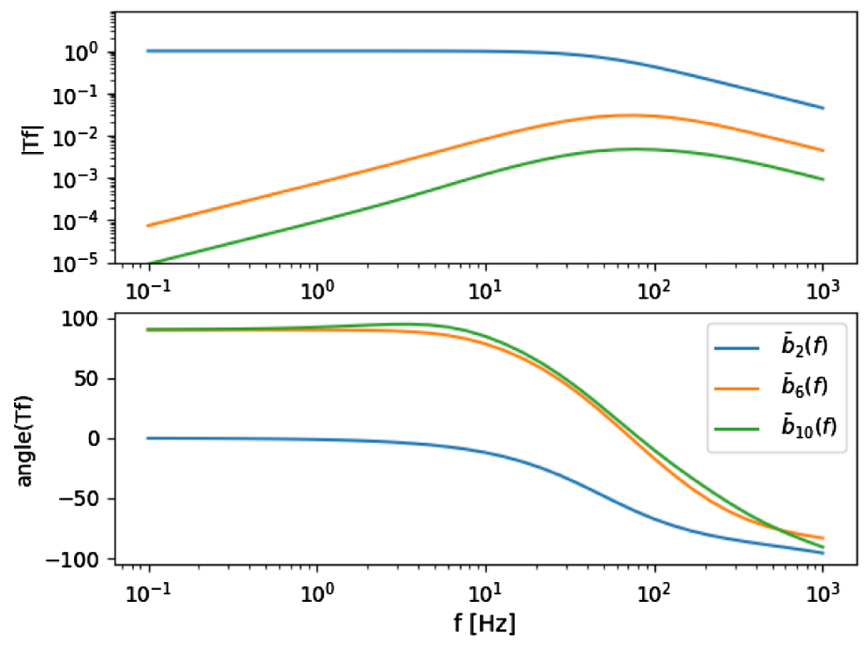

(b) LHC Main Quadrupole at $20 \mathrm{~K}$.

FIG. 14. AC multipole analysis for the beam screens of the LHC Main Dipoles.

\section{A. LHC quadrupole with position dependent magneto-resistance}

Expression (34) can be generalized by considering that all the quantities involved depend on $\theta$. For the specific case of LHC quadrupoles $(n=2)$ the simplified expression in (38) can still be considered valid within angle $\alpha$, whereas within angle $\beta$ it is reasonable to assume that only the conductivity $\sigma$ varies with the $\theta$ as the magnitude B of
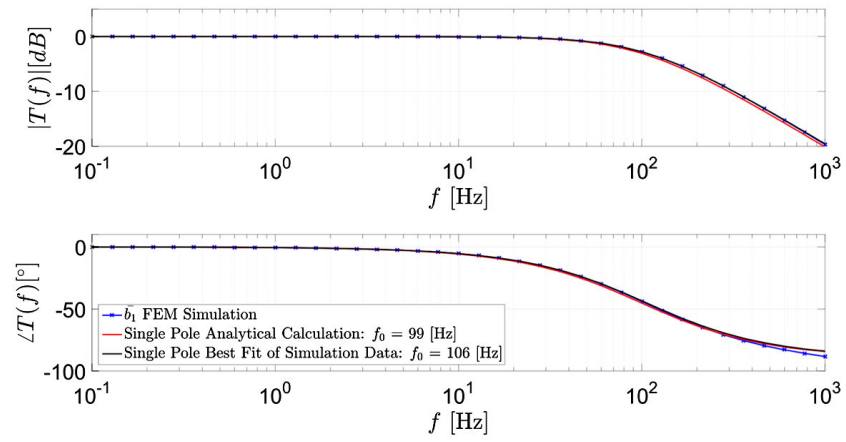

(a) LHC Main Dipoles at $20 \mathrm{~K}$
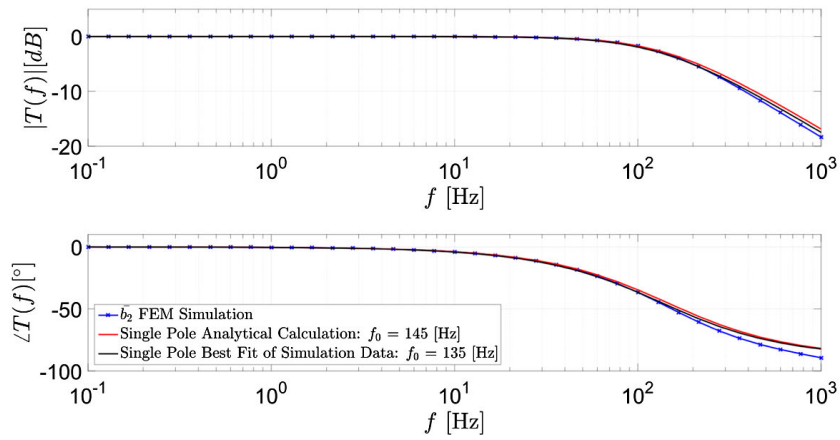

(b) LHC Main Quadrupoles at $20 \mathrm{~K}$

FIG. 15. Frequency response of shielding effect inside the LHC main dipoles. magnetic induction field is constant within angle $\alpha$. These considerations are summarized in the following equation:

$$
\begin{aligned}
\hat{A \sigma_{B S}} & =\frac{\pi}{2} \int_{0}^{2 \pi} \bar{\rho}_{0}(\theta) \Delta(\theta) \sigma(\theta)|\cos 2 \theta| d \theta \\
& =4 \frac{\pi}{2} \int_{0}^{\frac{\pi}{2}} \bar{\rho}_{0}(\theta) \Delta(\theta) \sigma(\theta)|\cos 2 \theta| d \theta \\
& \approx 2 \pi\left[c_{\alpha}^{(2)} \bar{\rho}_{\alpha}\left(\Delta \sigma_{\min }\right)_{\alpha}+F_{2 \beta} \bar{\rho}_{\beta} \Delta \int_{\alpha}^{\frac{\pi}{2}} \sigma(\theta)|\cos 2 \theta| d \theta\right] .
\end{aligned}
$$

The interpolated, position dependent, conductivity can be easily expressed as:

$$
\sigma(\theta)=\sigma_{\min }-\frac{\sigma_{\max }-\sigma_{\min }}{B_{\max }-B_{\min }}\left(\frac{B_{\min }}{\sin \theta}-B_{\max }\right),
$$

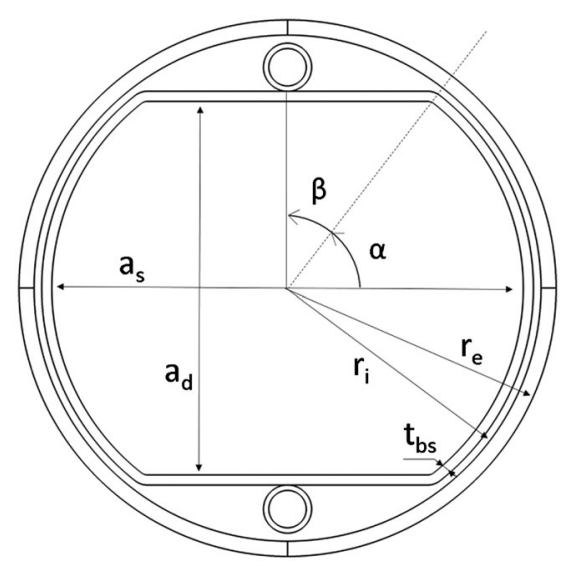

FIG. 16. LHC beam screen cross section. 

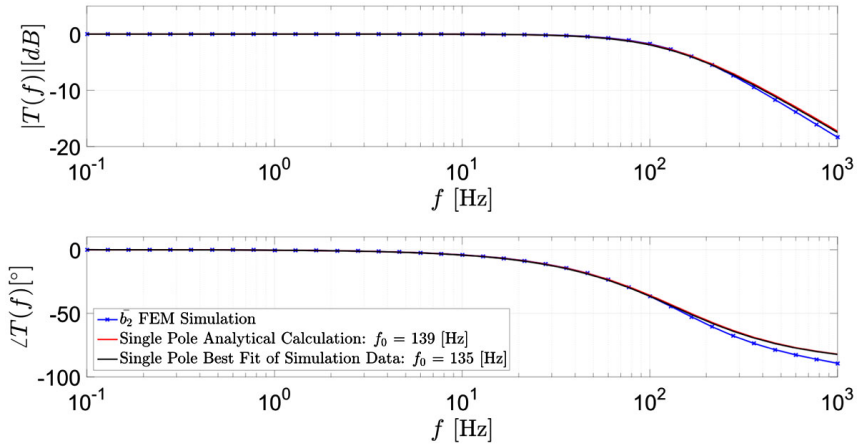

FIG. 17. LHC main quadrupoles at $20 \mathrm{~K}$ with position dependent magnetoresistance.

where $\alpha \leq \theta \leq \frac{\pi}{2}, \mathrm{~B}_{\max }=\mathrm{B}(\alpha), \mathrm{B}_{\min }=\mathrm{B}\left(\frac{\pi}{2}\right), \sigma_{\max }=\sigma\left(\frac{\pi}{2}\right)$ and $\sigma_{\min }=\sigma(\alpha)$. Indeed the magnitude of the magnetic induction field goes as $1 / \sin \theta$ for $\alpha \leq \theta \leq \pi / 2$. The interpolation is based on the assumption that at high field the decrease of conductivity is linear with the magnitude of the field [30].

The values used are reported in the last two rows of Table III. With this more accurate approximation the estimation of the cutoff frequency can be improved as shown in Fig. 17 where the estimation error drops from $+7.4 \%(145 \mathrm{~Hz})$ down to $+3 \%(139 \mathrm{~Hz})$ with respect to the fitted value of $135 \mathrm{~Hz}$.

\section{CONCLUSION AND FUTURE WORK}

The magnetic transfer function of a beam screen has been rigorously defined according to the magnet type. Specific 2D FEM simulations in the frequency domain have been performed for different HL-LHC beam screen layouts and validated against those evaluated in the time domain. All simulations were performed at the maximum tolerated temperature and including magnetoresistive effects, which represent the worst case scenarios in terms of a current ripple being transferred to the magnetic field and then affecting the beam. The postprocessing technique proposed in this paper allowed the evaluation and comparison of the frequency response of the HL-LHC beam screen configurations as well as those of LHC main dipoles and quadrupoles. An easy-to-use approximated formula for the single pole cutoff frequency has also been generalized for the case of a multilayer beam screen and noncylindrical geometry which well matches the simulation on the considered geometries. Applying the formula to noncylindrical geometry is an approximation, with generally unknown accuracy. Once validated for a class of geometries, the formula can be used to quickly assess the cutoff frequency at different temperatures or the impact of different materials without requiring a complete simulation and the subsequent postprocessing. Furthermore, the proposed description is deemed to fully characterize $T_{\mathrm{B}_{\mathrm{m}}}$ to $B_{\mathrm{b}}(f)$. As such, it is considered an important contribution for the specification of power converter performance for the HL-LHC. Future work will focus on the characterization of the full transfer function from power converter voltage to the magnetic field applied to the particles.

\section{ACKNOWLEDGMENTS}

Research supported by the HL-LHC project. This manuscript has been coauthored by Fermi Research Alliance, LLC under Contract No. DE-AC02-07CH11359 with the U.S. Department of Energy, Office of Science, Office of High Energy Physics. The authors are grateful to Sergio Calatroni (CERN) and Massimo Giovannozzi (CERN) for their insightful contributions.

\section{APPENDIX: IDEAL THIN CONDUCTIVE SHELL INSIDE A PURE MONOPOLE}

Following closely the analysis conducted in [16], and using the same notation, an oscillating current sheet at radius $r=b$ with frequency $f$ gives a vector potential for $r<b$ :

$$
A_{z}(r, \theta, f)=\mu_{0} \frac{b}{2 n}\left(\frac{r}{b}\right)^{n} J_{z}(b, \theta, f),
$$

where, for an ideal $n$-pole field:

$$
J_{z}(b, \theta, f)=\frac{N_{c} I_{o}}{4 b} \cos (n \theta) \sin (2 \pi f t) .
$$

The dependence on $t$ will be omitted in the following.

If at $r=a(a<b)$ there is an infinitely thin sheet of conductor, a current is induced which can be written as:

$J_{z}^{\prime}(a, \theta, f)=\sigma_{s} E_{z}=-j 2 \pi f \sigma_{s}\left[A_{z}(a, \theta, f)+A_{z}^{\prime}(a, \theta, f)\right]$,

where $\sigma_{s}$ is the surface conductivity of the sheet and $A_{z}+A_{z}^{\prime}$ represents the total vector potential $A_{z}^{\text {tot }} . A_{z}^{\prime}$ can be expressed, for $r \leq a$, considering the new source $J_{z}^{\prime}(a, \theta, f)$ as:

$$
\begin{aligned}
A_{z}^{\prime}(r, \theta, f) & =\mu_{0} \frac{a}{2 n}\left(\frac{r}{a}\right)^{n} J_{z}^{\prime}(a, \theta, f) \\
& =-j 2 \pi f \sigma_{s} \mu_{0} \frac{a}{2 n}\left(\frac{r}{a}\right)^{n}\left[A_{z}(a, \theta, f)+A_{z}^{\prime}(a, \theta, f)\right] \\
& =-j 2 \pi f \sigma_{s} \mu_{0} \frac{a}{2 n}\left[A_{z}(r, \theta, f)+A_{z}^{\prime}(r, \theta, f)\right] .
\end{aligned}
$$

Now $A_{z}^{\prime}$ can be expressed in terms of $A_{z}$ as follows:

$$
A_{z}^{\prime}(r, \theta, f)=-\frac{j 2 \pi f \sigma_{s} \mu_{0} \frac{a}{2 n}}{1+j 2 \pi f \sigma_{s} \mu_{0} \frac{a}{2 n}} A_{z}(r, \theta, f),
$$


and finally:

$$
A_{z}^{\mathrm{tot}}(r, \theta, f)=A_{z}(r, \theta, f)+A_{z}^{\prime}(r, \theta, f)=\frac{A_{z}(r, \theta, f)}{1+j 2 \pi f \sigma_{s} \mu_{0} \frac{a}{2 n}} .
$$

Therefore the conductive sheet at $r=a$ introduces a pole in the transfer function of the vector potential with a cutoff frequency:

$$
f_{0}=\frac{2 n}{\mu_{0} 2 \pi a \sigma_{s}}
$$

[1] L. Rossi and O. Brüning, The high luminosity large hadron collider. The new machine for illuminating the mysteries of universe, Advanced Series on Directions in High Energy Physics (World Scientific, Singapore, 2015), Vol. 24.

[2] G. Apollinari, I. Bejar Alonso, O. Brüning, P. Fessia, M. Lamont, L. Rossi, and L. Tavian, High-Luminosity Large Hadron Collider (HL-LHC), CERN Yellow Reports: Monographs, CERN, 2017, Vol. 4, http://dx.doi.org/ 10.23731/CYRM-2017-004.

[3] R. Kersevan, C. Garion, and N. Kos, Preliminary design of the Hilumi-LHC triplet area beam screen, Technical Report No. CERN-ACC-2014-0268, 2014.

[4] X. Altuna et al., The 1991 dynamic aperture experiment at the CERN SPS, AIP Conf. Proc. 255, 355 (1992).

[5] W. Fischer, F. Schmidt, and M. Giovannozzi, Dynamic aperture experiment at a synchrotron, Phys. Rev. E 55, 3507 (1997).

[6] P. Burla, D. Cornuet, K. Fischer, P. Leclere, and F. Schmidt, Power supply ripple study at the SPS, AIP Conf. Proc. 326, 167 (1995).

[7] L. R. Evans, The beam-beam interaction, Report No. CERN-SPS-83-38-DI-MST, 1984, p. 49.

[8] O. S. Brüning, Diffusion in a FODO cell due to modulation effects in the presence of nonlinear fields, Part. Accel. 41, 133 (1992).

[9] O. S. Brüning and F. Willeke, Reduction of Proton Losses in HERA by Compensating Tune Ripple due to Power Supplies, Phys. Rev. Lett. 76, 3719 (1996).

[10] D. Neuffer, A. Riddiford, and A. G. Ruggiero, Study of the periodic tune modulation with the beam-beam effect, IEEE Trans. Nucl. Sci. 30, 2433 (1983).

[11] O. S. Brüning, P. Collier, P. Lebrun, S. Myers, R. Ostojic, J. Poole, and P. Proudlock, LHC Design Report, CERN Yellow Report No. CERN-2004-003-V-1: Monographs (CERN, Geneva, 2004), https://cds.cern.ch/record/782076.

[12] M. Fitterer, R. De Maria, S. Fartoukh, and M. Giovannozzi, Beam dynamics requirements for the powering scheme of the HL-LHC triplet, in Proceedings, 6th International Particle Accelerator Conference (IPAC 2015): Richmond, Virginia, USA, 2015 (2015), p. TUPTY035, http:// accelconf.web.cern.ch/AccelConf/IPAC2015/papers/ tupty035.pdf.
[13] M. Fitterer, R. De Maria, S. Fartoukh, and M. Giovannozzi, Powering schemes for inner triplet, Q4 and D1/D2, 21st HL-LHC Technical Committee, 2015, https://indico.cern .ch/event/373539/.

[14] M. Fitterer, R. De Maria, S. Fartoukh, and M. Giovannozzi, Follow up of powering schemes for IT, Q4 and D1/D2, 22nd HL-LHC Technical Committee, 2015, https://indico .cern.ch/event/373541/.

[15] M. Fitterer and R. De Maria, Progress on power converter ripple effects and specifications for HL-LHC, 2014, https:// ab-dep-abp.web.cern.ch/ab-dep-abp/HSS/HSS_meetings/ 2014/20140217/HSSMeeting 17022014.pdf.

[16] R. Shafer, Eddy currents, dispersion relations, and transient effects in superconducting magnets, Technical Report No. TM-991, 1980, http://inspirehep.net/record/156969/ files/fermilab-tm-0991.PDF.

[17] B. Podobedov, L. Ecker, D. Harder, and R. George, Eddy current shielding by electrically thick vacuum chambers, in Proceedings of the 23rd Particle Accelerator Conference, Vancouver, Canada, 2009 (IEEE, Piscataway, NJ, 2009), pp. 3398-3400.

[18] S. Lee, A multipole expansion for the field of vacuum chamber eddy currents, Nucl. Instrum. Methods Phys. Res., Sect. A 300, 151 (1991).

[19] F. Zimmermann, Emittance growth and proton beam lifetime in HERA, Ph.D. dissertation, Hamburg U., 1993, http://inspirehep.net/record/354381/files/354381.pdf.

[20] S. Sgobba, Materials for high vacuum technology, an overview, CERN Technical Report No. CERN-TS-2006004, 2006.

[21] E. Todesco, H. Allain, G. Ambrosio, G. Arduini, F. Cerutti, R. De Maria, L. Esposito, S. Fartoukh, P. Ferracin, H. Felice et al., A first baseline for the magnets in the high luminosity lhc insertion regions, IEEE Trans. Appl. Supercond. 24, 1 (2014).

[22] C. Garion, R. Kersevan, and V. Baglin, Preliminary design of the High-Luminosity LHC beam screen with shielding, Report No. CERN-ACC-2014-0268, 2014.

[23] V. Baglin, L. Tavian, P. Lebrun, and R. van Weelderen, Cryogenic beam screens for high-energy particle accelerators, Report No. CERN-ATS-2013-006, 2013.

[24] Comsol multiphysics software, https://www.comsol.com, 2017.

[25] M. Morrone, C. Garion, M. Aurisicchio, and P. Chiggiato, Mechanical design of the High Luminosity Large Hadron Collider (HL-LHC) beam screen through a multiphysics model, Proc. Sci., ICHEP2016 (2016) 914.

[26] Z. Zeng, L. Udpa, S. S. Udpa, and M. S. C. Chan, Reduced magnetic vector potential formulation in the finite element analysis of eddy current nondestructive testing, IEEE Trans. Magn. 45, 964 (2009).

[27] AC/DC Module-User's Guide, COMSOL AB, 2016.

[28] J. G. Hust and A. B. Lankford, Thermal conductivity of aluminum, copper, iron, and tungsten for temperatures from $1 \mathrm{~K}$ to the melting point National Bureau of Standards Technical Report No. NBSIR 84-3007, 1984, https://ia800400.us.archive.org/34/items/ thermalconducti8430hust_0/thermalconducti8430hust_0 .pdf. 
[29] E. Drexler, N. Simon, and R. Reed, Properties of copper and copper alloys at cryogenic temperatures, NIST, Technical Report No. NIST-MN-177, 1992.

[30] E. Métral, Beam screen issues, arXiv:1108.1643; CERN Yellow Report No. CERN-2011-003, pp. 83-89, http://cds .cern.ch/record/1373989.

[31] P. R. Amestoy, I. S. Duff, and J.-Y. L'excellent, Multifrontal parallel distributed symmetric and unsymmetric solvers, Comput. Methods Appl. Mech. Eng. 184, 501 (2000).

[32] M. Morrone, C. Garion, M. Aurisicchio, and P. Chiggiato, A coupled multiphysics fem model to investigate electromagnetic, thermal and mechanical effects in complex assemblies: The design of the high-luminosity large hadron collider beam screen, Appl. Math. Model. 57, 280 (2018).
[33] S. Russenschuck, Field Computation for Accelerator Magnets: Analytical and Numerical Methods for Electromagnetic Design and Optimization (Wiley, New York, 2010), http://cds.cern.ch/record/1221810.

[34] S. Celozzi, R. Araneo, and G. Lovat, Electromagnetic Shielding (Wiley-IEEE Press, New York, 2008).

[35] A. Clark, G. Childs, and G. Wallace, Electrical resistivity of some engineering alloys at low temperatures, Cryogenics 10, 295 (1970).

[36] C. Garion, L. Dufay-Chanat, T. Koettig, W. Machiocha, and M. Morrone, Material characterisation and preliminary mechanical design for the HL-LHC shielded beam screens operating at cryogenic temperatures, in IOP Conference Series: Materials Science and Engineering (IOP Publishing, Tucson, 2015), Vol. 102, p. 012013, DOI: 10.1088/ $1757-899 X / 102 / 1 / 012013$. 